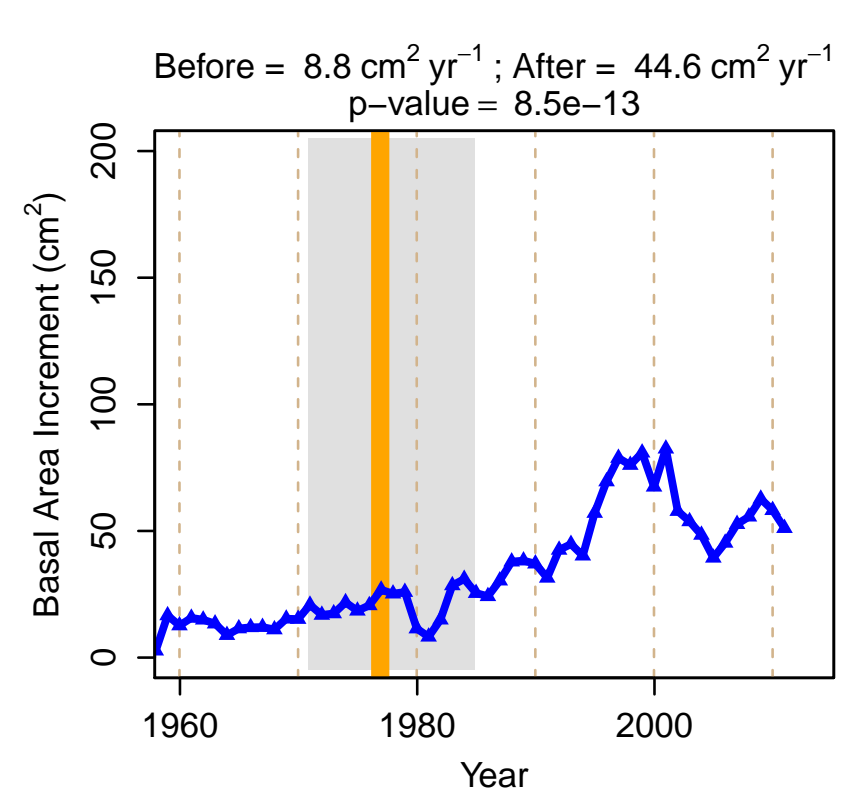

\section{Strong Response}

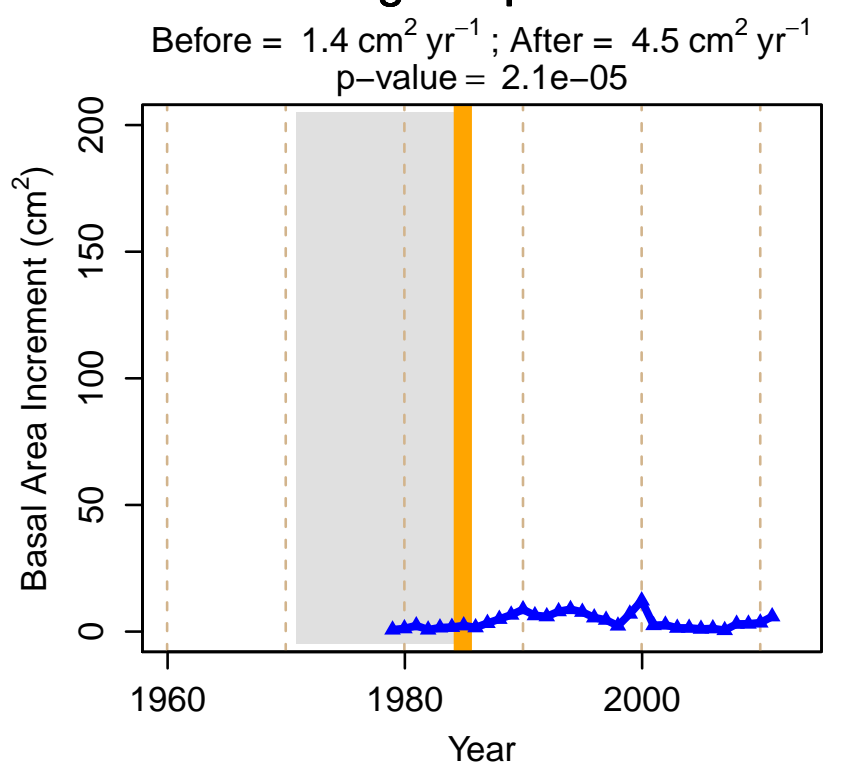

Before $=29.2 \mathrm{~cm}^{2} \mathrm{yr}^{-1} ;$ After $=108 \mathrm{~cm}^{2} \mathrm{yr}^{-1}$

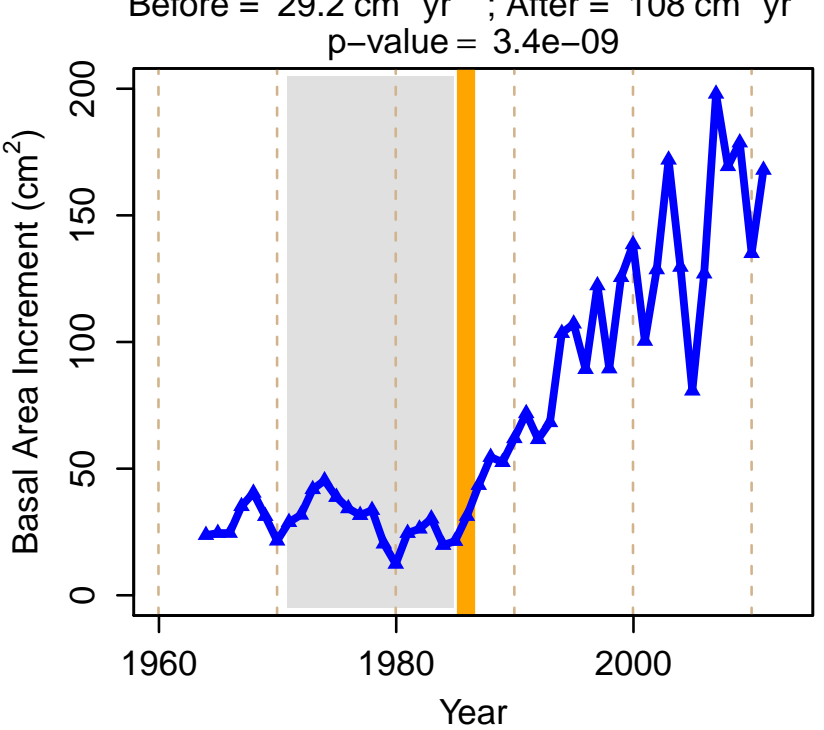

Before $=20.9 \mathrm{~cm}^{2} \mathrm{yr}^{-1} ;$ After $=70.4 \mathrm{~cm}^{2} \mathrm{yr}^{-1}$

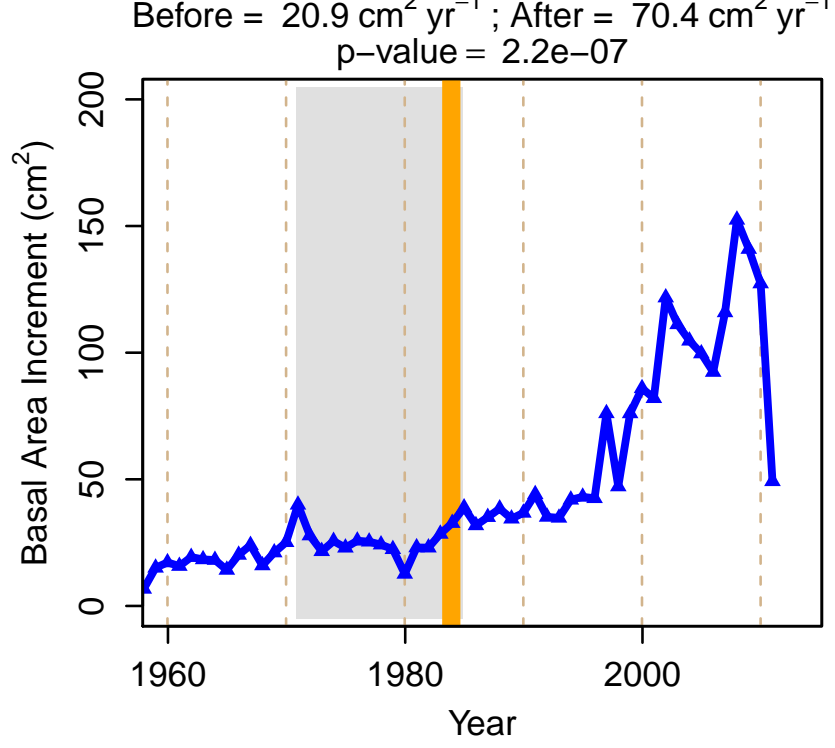

Before $=10.1 \mathrm{~cm}^{2} \mathrm{yr}^{-1} ;$ After $=30.8 \mathrm{~cm}^{2} \mathrm{yr}^{-1}$

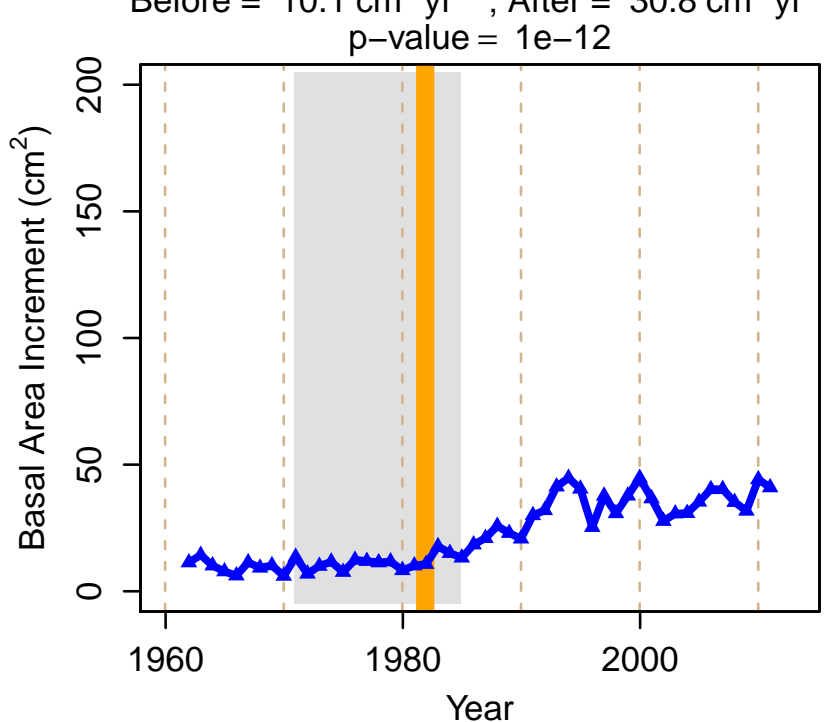

Before $=7.5 \mathrm{~cm}^{2} \mathrm{yr}^{-1} ;$ After $=32.7 \mathrm{~cm}^{2} \mathrm{yr}^{-1}$

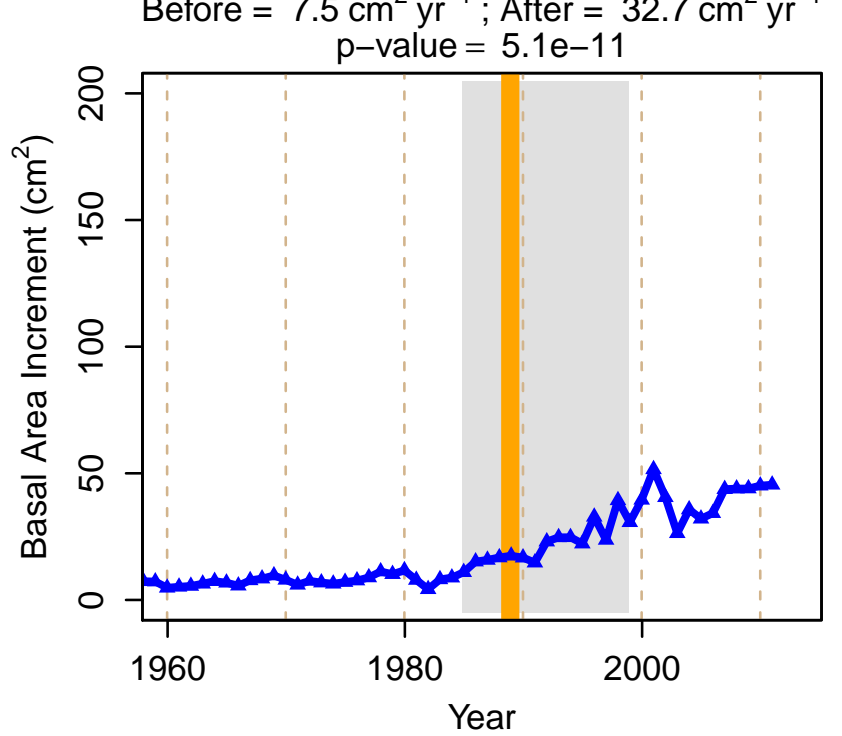

Before $=5.3 \mathrm{~cm}^{2} \mathrm{yr}^{-1} ;$ After $=29.5 \mathrm{~cm}^{2} \mathrm{yr}^{-1}$ $\mathrm{p}-$ value $=5.1 \mathrm{e}-11$

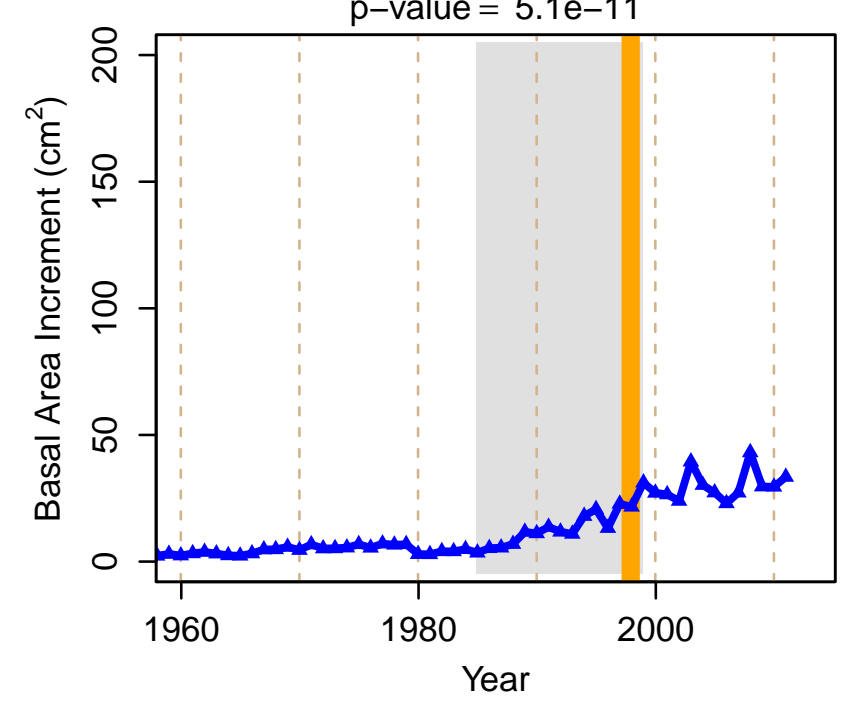

Before $=3.8 \mathrm{~cm}^{2} \mathrm{yr}^{-1} ;$ After $=18.5 \mathrm{~cm}^{2} \mathrm{yr}^{-1}$ $\mathrm{p}-$ value $=5.8 \mathrm{e}-12$
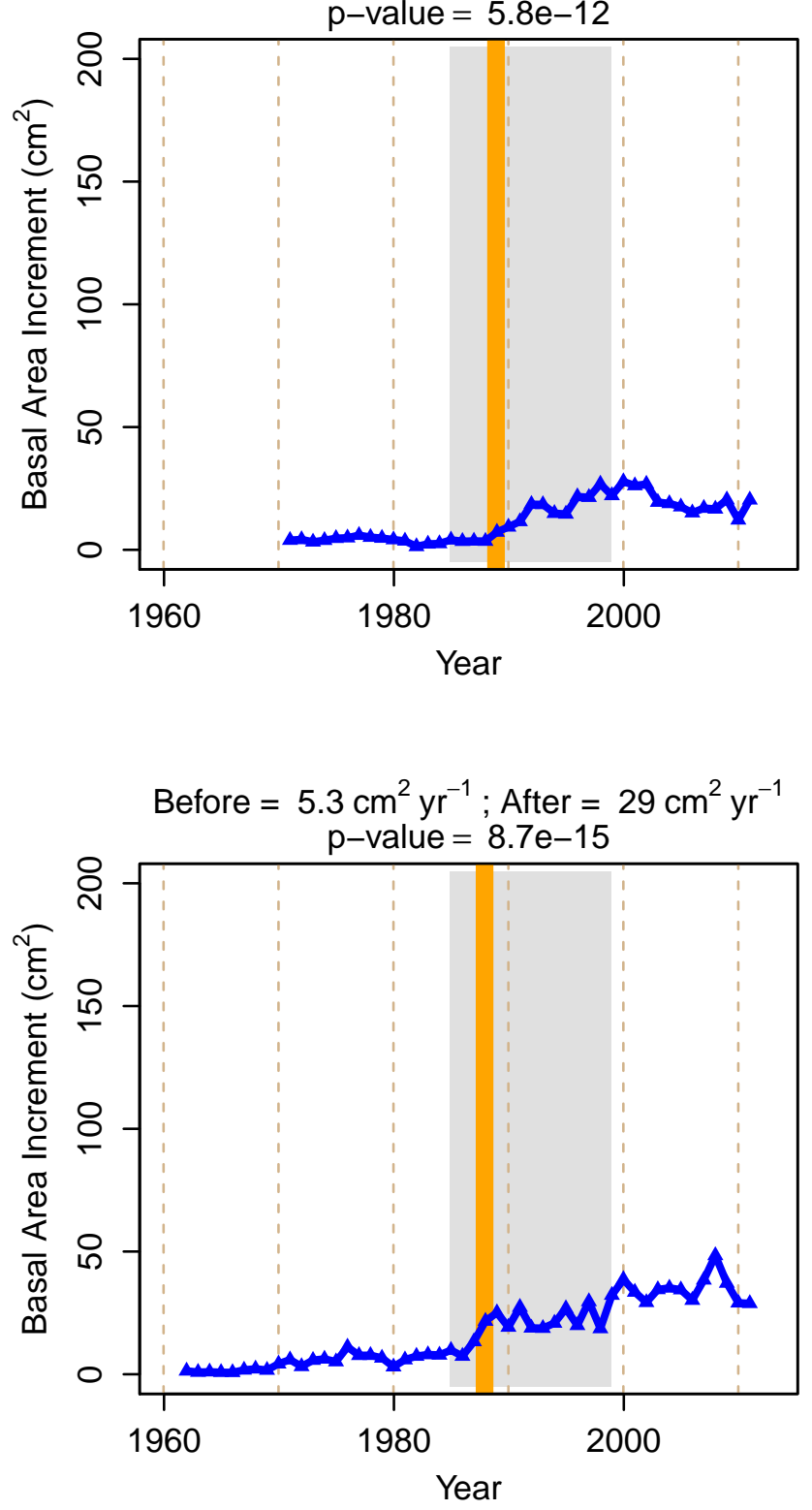


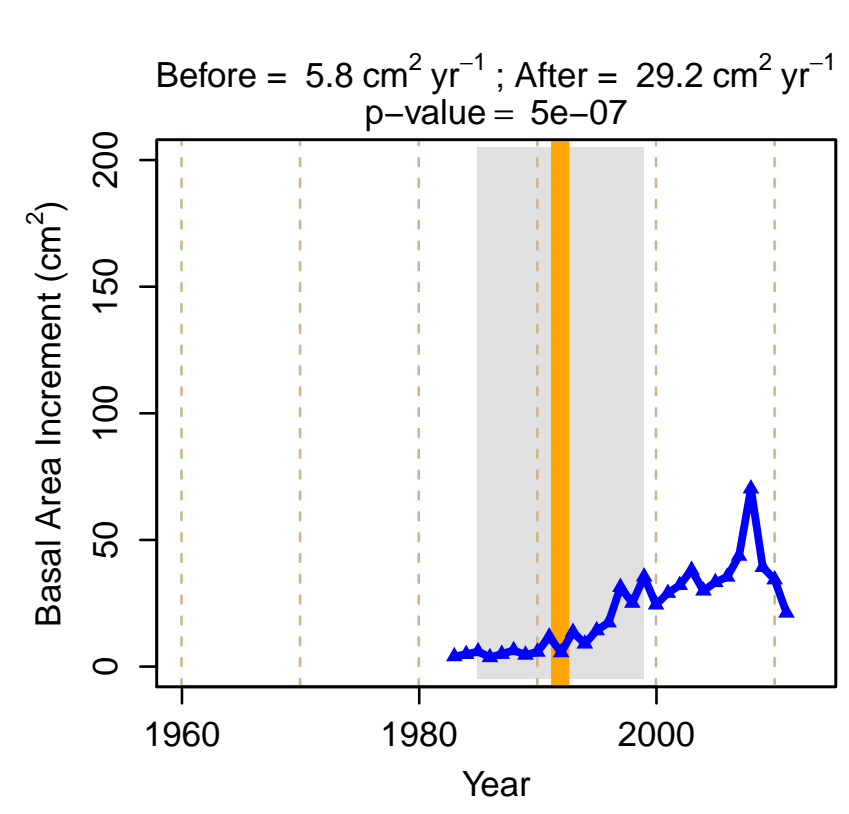

\section{Strong Response}

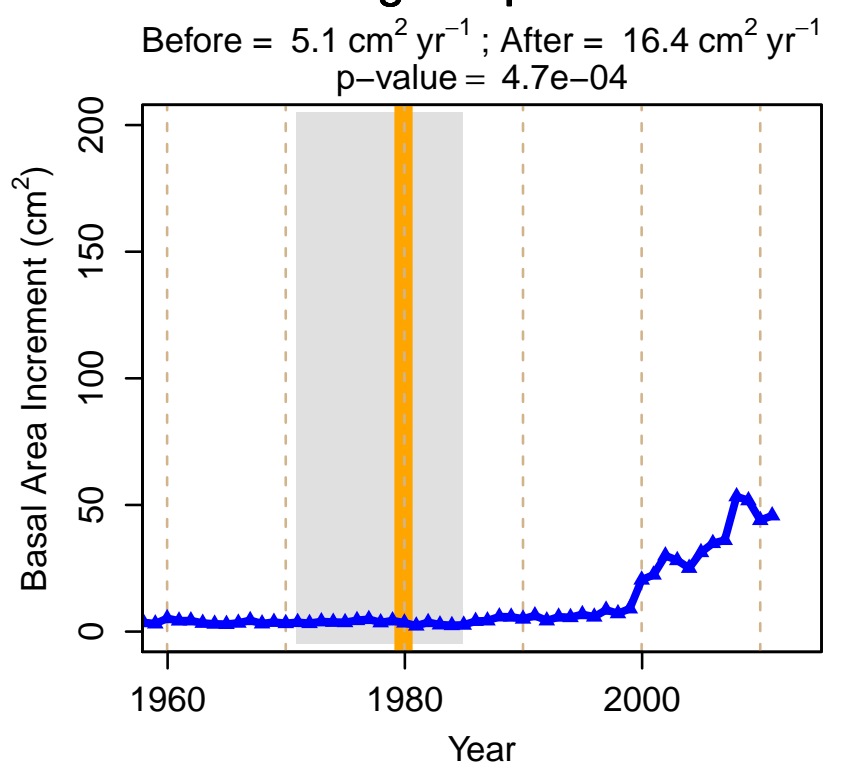

Before $=15.9 \mathrm{~cm}^{2} \mathrm{yr}^{-1} ;$ After $=48.3 \mathrm{~cm}^{2} \mathrm{yr}^{-1}$ $\mathrm{p}-$ value $=9.1 \mathrm{e}-04$
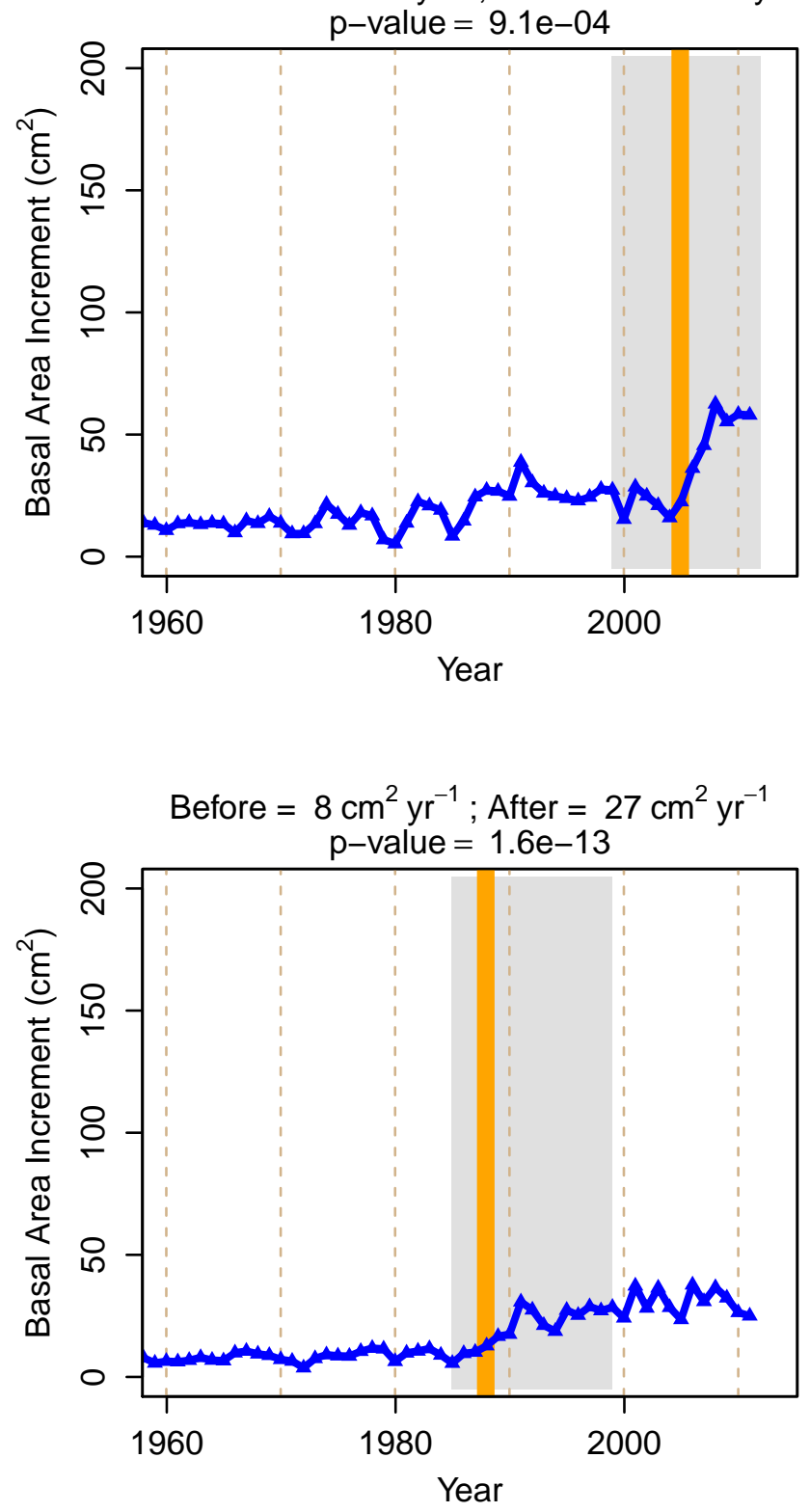

Before $=1.6 \mathrm{~cm}^{2} \mathrm{yr}^{-1} ;$ After $=29.9 \mathrm{~cm}^{2} \mathrm{yr}^{-1}$

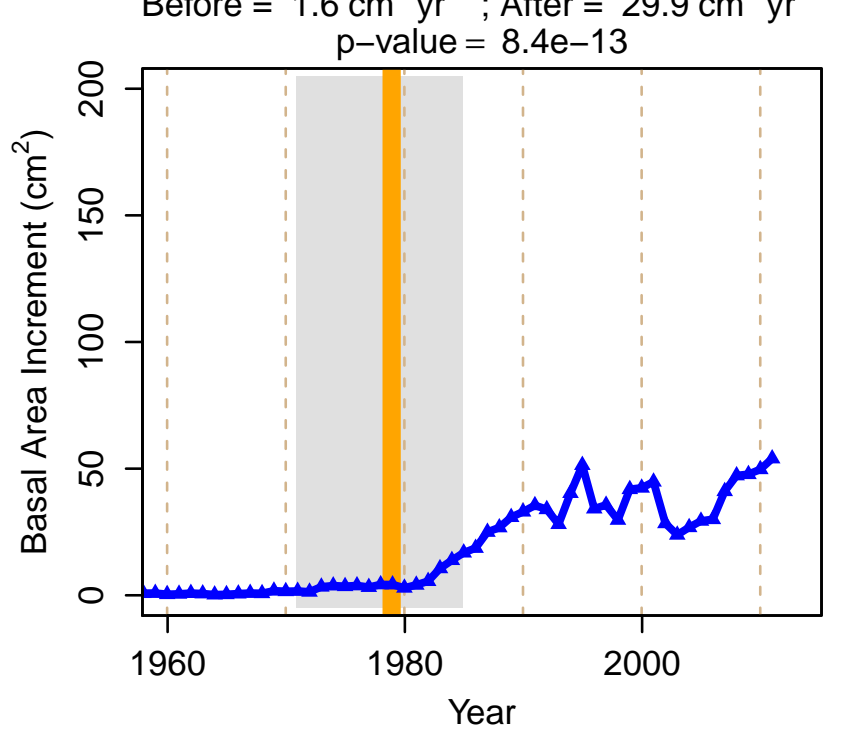

Before $=10.9 \mathrm{~cm}^{2} \mathrm{yr}^{-1} ;$ After $=51.2 \mathrm{~cm}^{2} \mathrm{yr}^{-1}$

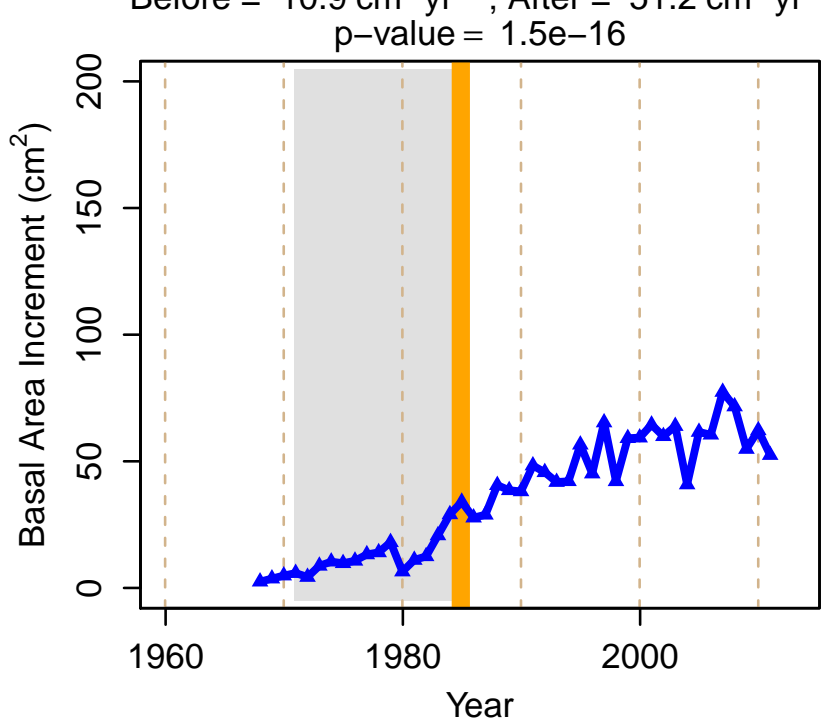

Before $=21.1 \mathrm{~cm}^{2} \mathrm{yr}^{-1} ;$ After $=73.5 \mathrm{~cm}^{2} \mathrm{yr}^{-1}$ $\mathrm{p}-$ value $=8.1 \mathrm{e}-07$

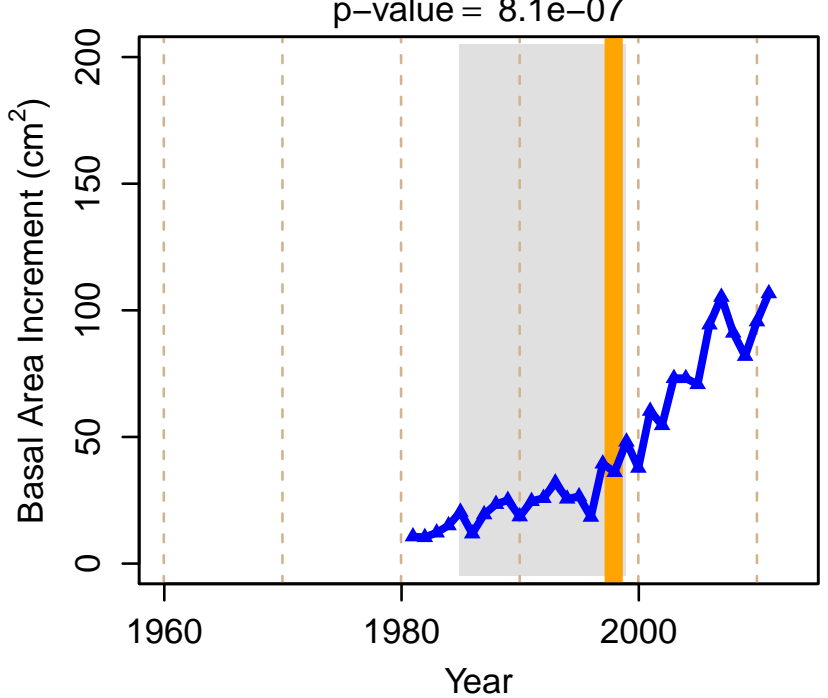

Before $=6.5 \mathrm{~cm}^{2} \mathrm{yr}^{-1} ;$ After $=46.9 \mathrm{~cm}^{2} \mathrm{yr}^{-1}$ $\mathrm{p}-$ value $=1.1 \mathrm{e}-03$

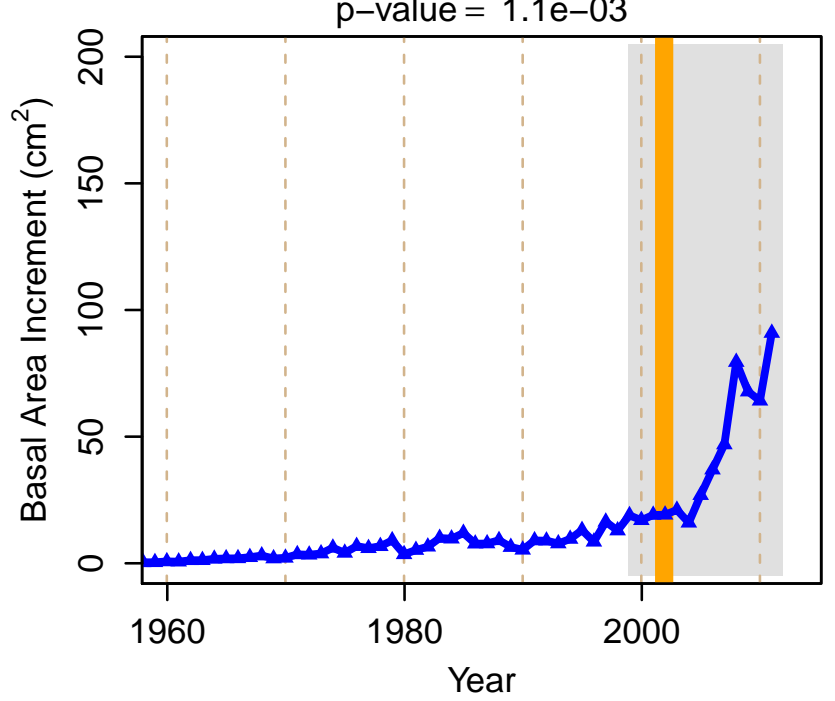

Before $=6.8 \mathrm{~cm}^{2} \mathrm{yr}^{-1} ;$ After $=27.2 \mathrm{~cm}^{2} \mathrm{yr}^{-1}$ $\mathrm{p}$-value $=4.9 \mathrm{e}-07$

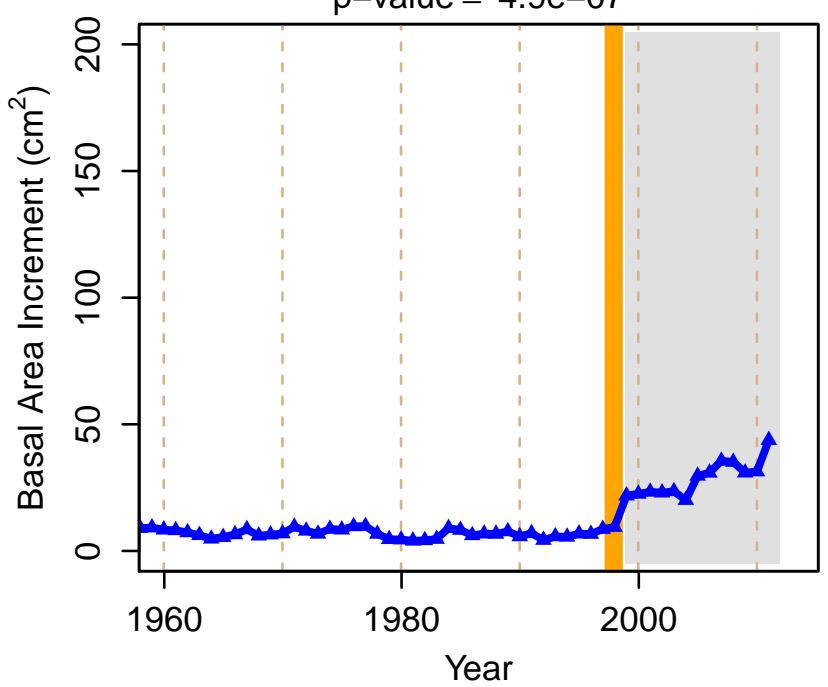




\section{Typical Response}
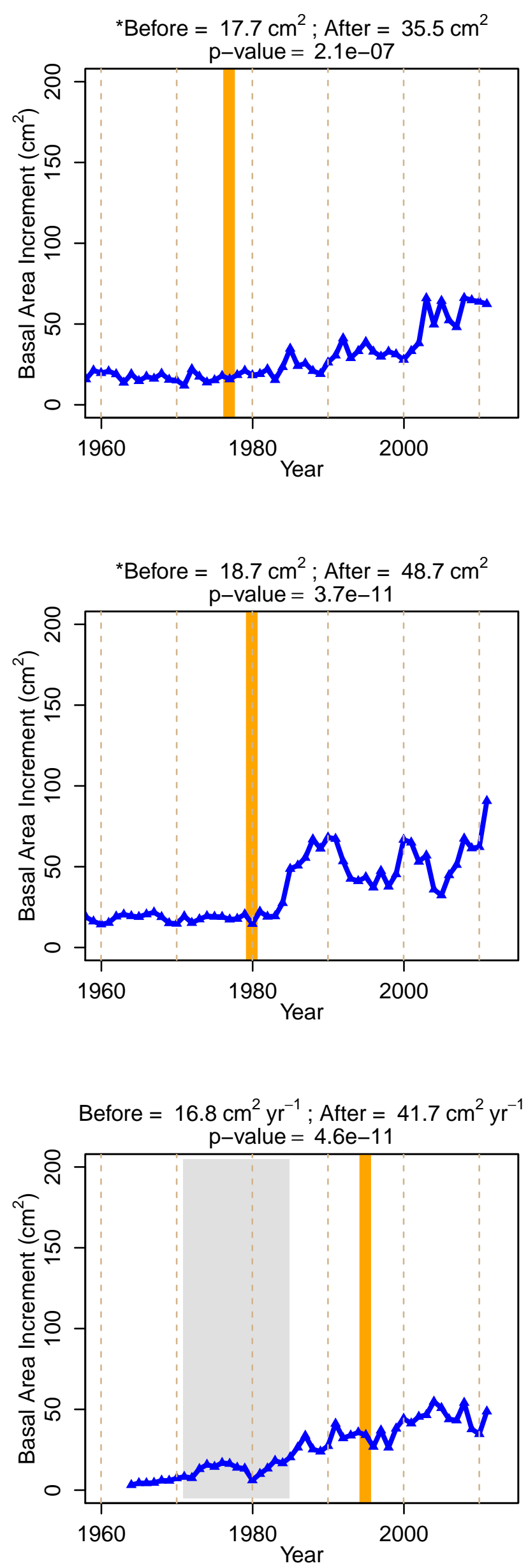

Before $=8.8 \mathrm{~cm}^{2} \mathrm{yr}^{-1} ;$ After $=16.6 \mathrm{~cm}^{2} \mathrm{yr}^{-1}$ $p-$ value $=4.3 \mathrm{e}-04$

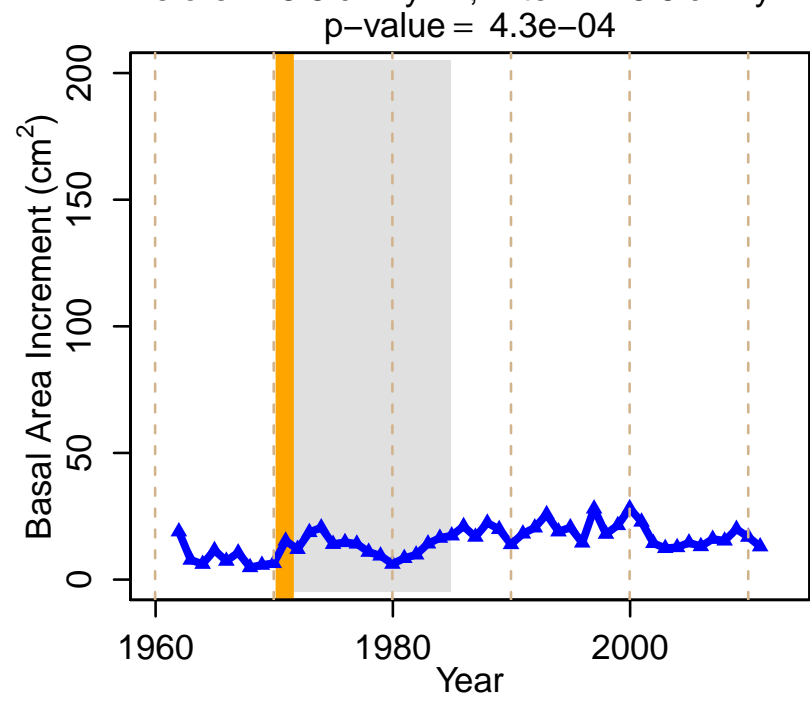

Before $=29.4 \mathrm{~cm}^{2} \mathrm{yr}^{-1} ;$ After $=62 \mathrm{~cm}^{2} \mathrm{yr}^{-1}$ $\mathrm{p}-$ value $=3.7 \mathrm{e}-04$

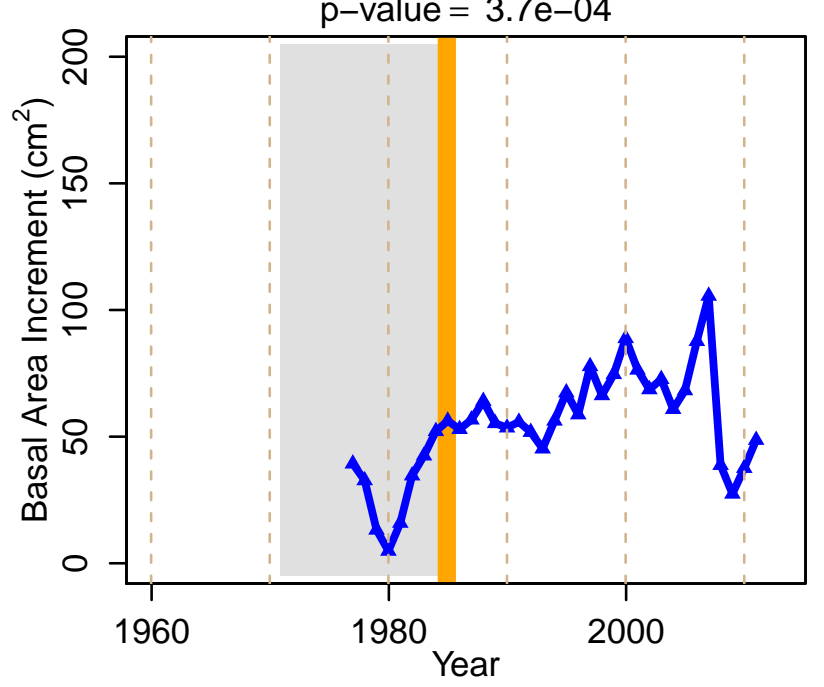

Before $=12 \mathrm{~cm}^{2} \mathrm{yr}^{-1} ;$ After $=16.6 \mathrm{~cm}^{2} \mathrm{yr}^{-1}$ $\mathrm{p}$-value $=1.4 \mathrm{e}-02$

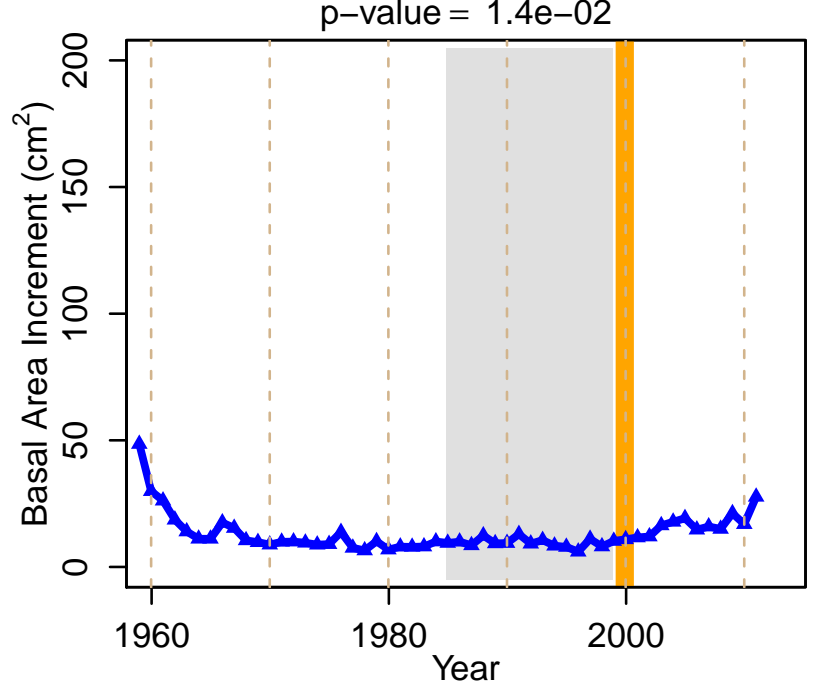

Before $=17.1 \mathrm{~cm}^{2} \mathrm{yr}^{-1} ;$ After $=32.8 \mathrm{~cm}^{2} \mathrm{yr}^{-1}$ $\mathrm{p}-$ value $=1.3 \mathrm{e}-07$

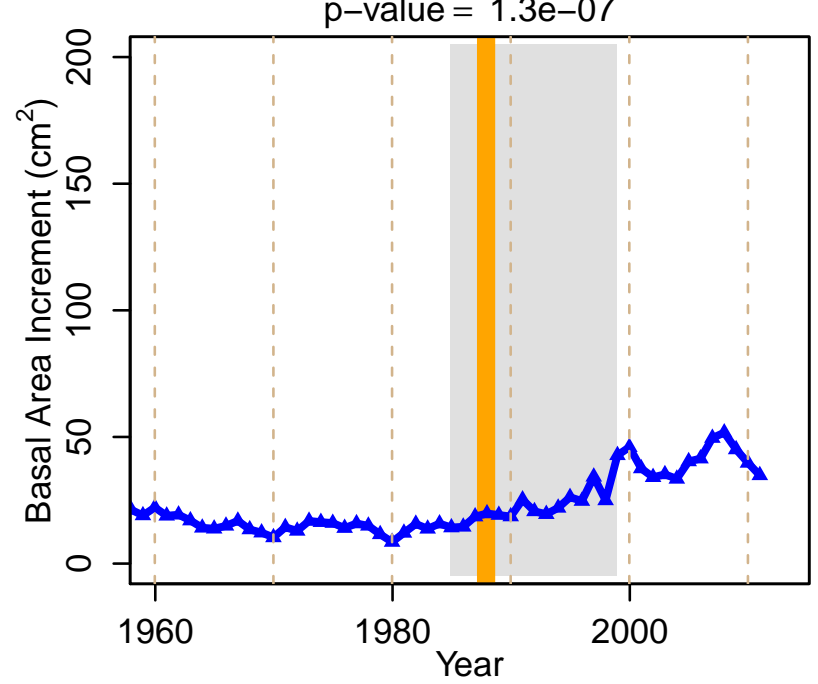

Before $=10.5 \mathrm{~cm}^{2} \mathrm{yr}^{-1} ;$ After $=18.8 \mathrm{~cm}^{2} \mathrm{yr}^{-1}$ $\mathrm{p}-$ value $=1.3 \mathrm{e}-03$

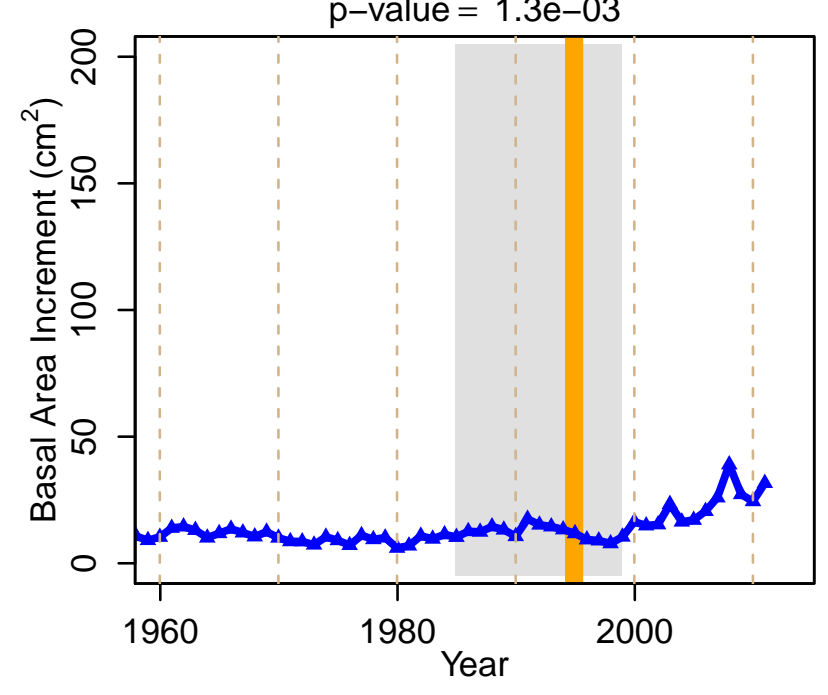

Before $=23.9 \mathrm{~cm}^{2} \mathrm{yr}^{-1} ;$ After $=35.7 \mathrm{~cm}^{2} \mathrm{yr}^{-1}$ $\mathrm{p}-$ value $=2.2 \mathrm{e}-07$

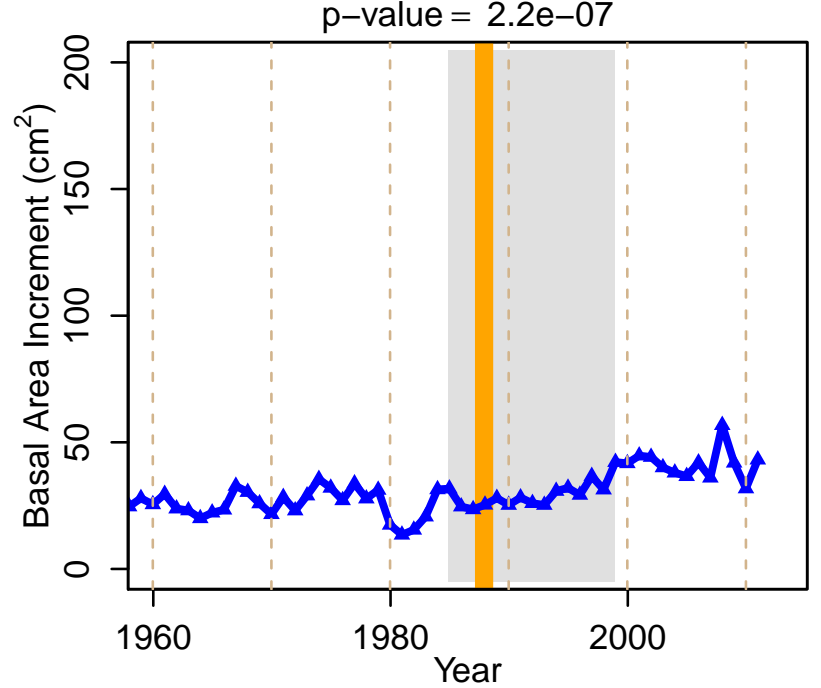




\section{Typical Response}

Before $=11.7 \mathrm{~cm}^{2} \mathrm{yr}^{-1} ;$ After $=27.8 \mathrm{~cm}^{2} \mathrm{yr}^{-1}$ $\mathrm{p}$-value $=6.1 \mathrm{e}-07$

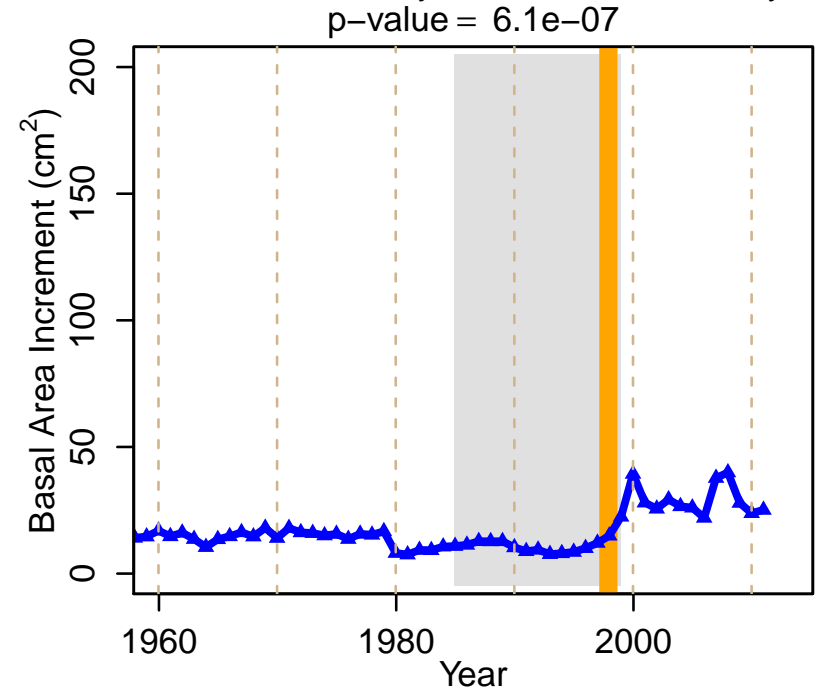

Before $=19.2 \mathrm{~cm}^{2} \mathrm{yr}^{-1} ;$ After $=33.9 \mathrm{~cm}^{2} \mathrm{yr}^{-1}$ $\mathrm{p}$-value $=3.1 \mathrm{e}-02$

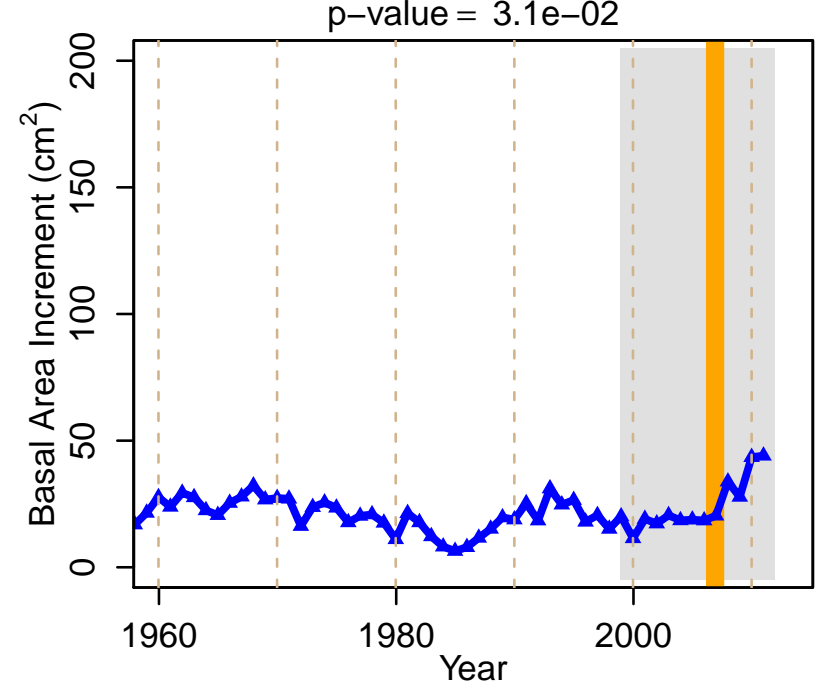

Before $=22.8 \mathrm{~cm}^{2} \mathrm{yr}^{-1} ;$ After $=54.6 \mathrm{~cm}^{2} \mathrm{yr}^{-1}$

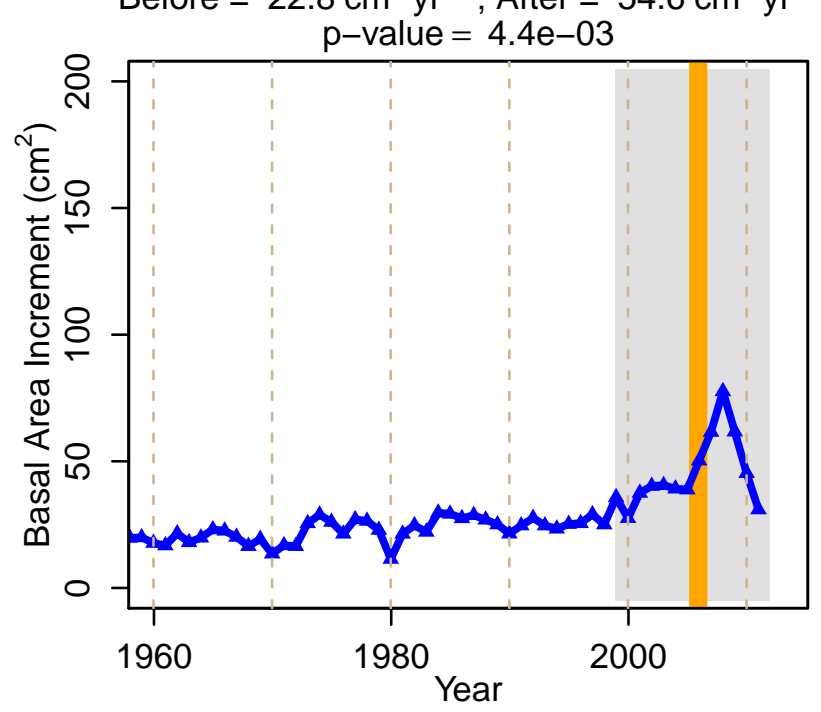

Before $=22 \mathrm{~cm}^{2} \mathrm{yr}^{-1} ;$ After $=39.2 \mathrm{~cm}^{2} \mathrm{yr}^{-1}$

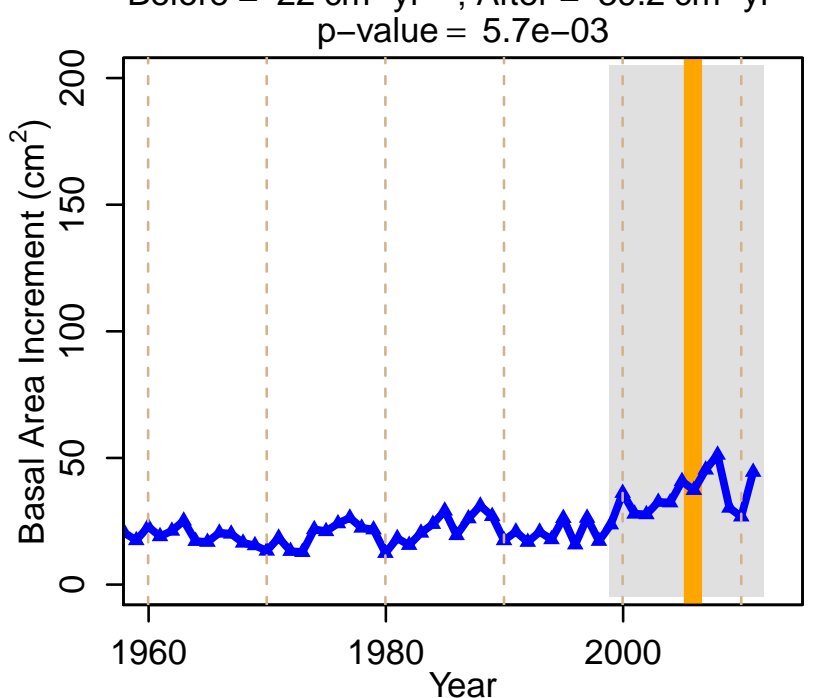

Before $=18.5 \mathrm{~cm}^{2} \mathrm{yr}^{-1} ;$ After $=32.1 \mathrm{~cm}^{2} \mathrm{yr}^{-1}$

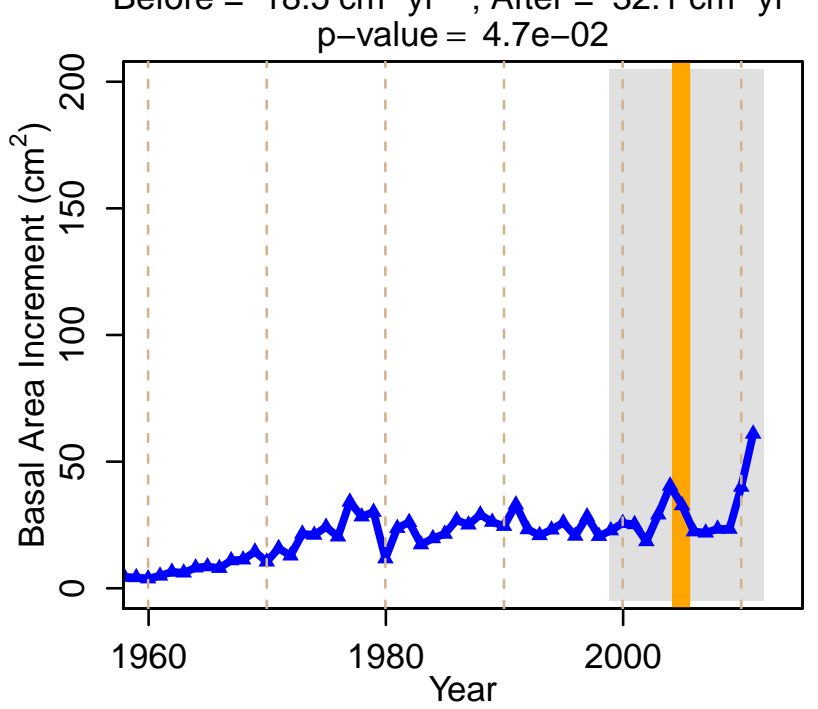

Before $=16.8 \mathrm{~cm}^{2} \mathrm{yr}^{-1} ;$ After $=39.8 \mathrm{~cm}^{2} \mathrm{yr}^{-1}$ $\mathrm{p}-$ value $=8.5 \mathrm{e}-03$

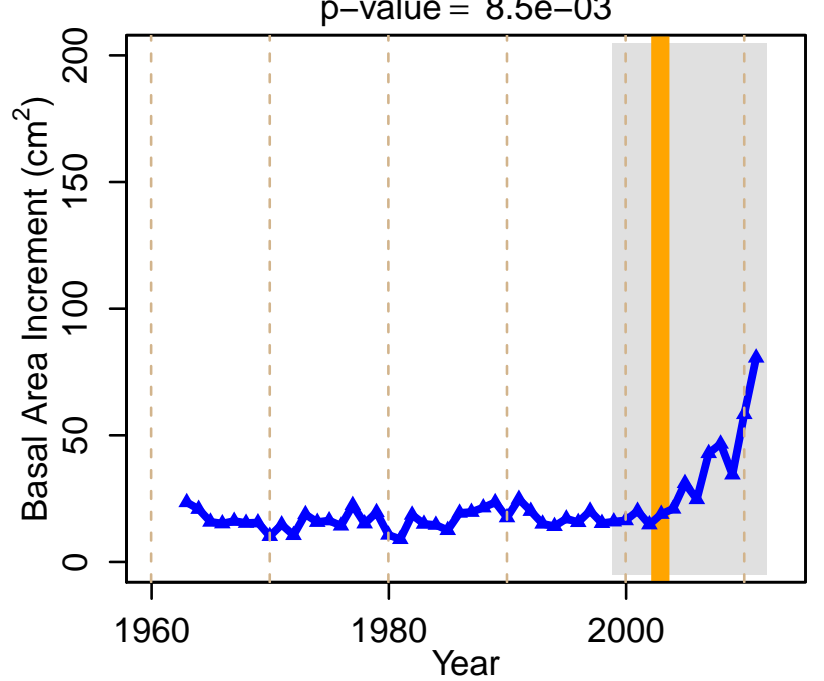

Before $=21.7 \mathrm{~cm}^{2} \mathrm{yr}^{-1} ;$ After $=30.5 \mathrm{~cm}^{2} \mathrm{yr}^{-1}$ $\mathrm{p}$-value $=1.2 \mathrm{e}-05$

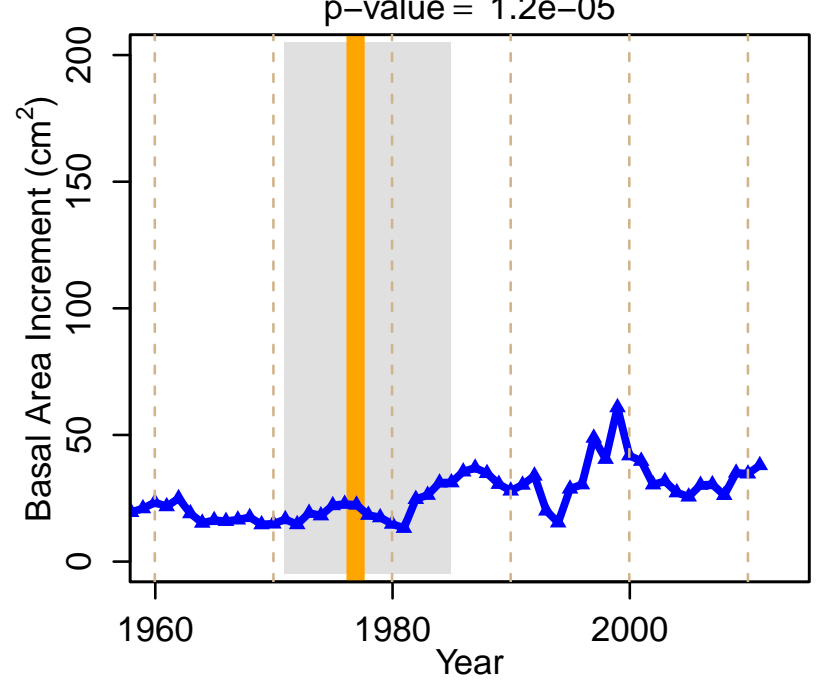

Before $=13.7 \mathrm{~cm}^{2} \mathrm{yr}^{-1} ;$ After $=20.3 \mathrm{~cm}^{2} \mathrm{yr}^{-1}$ $\mathrm{p}-$ value $=1.2 \mathrm{e}-05$

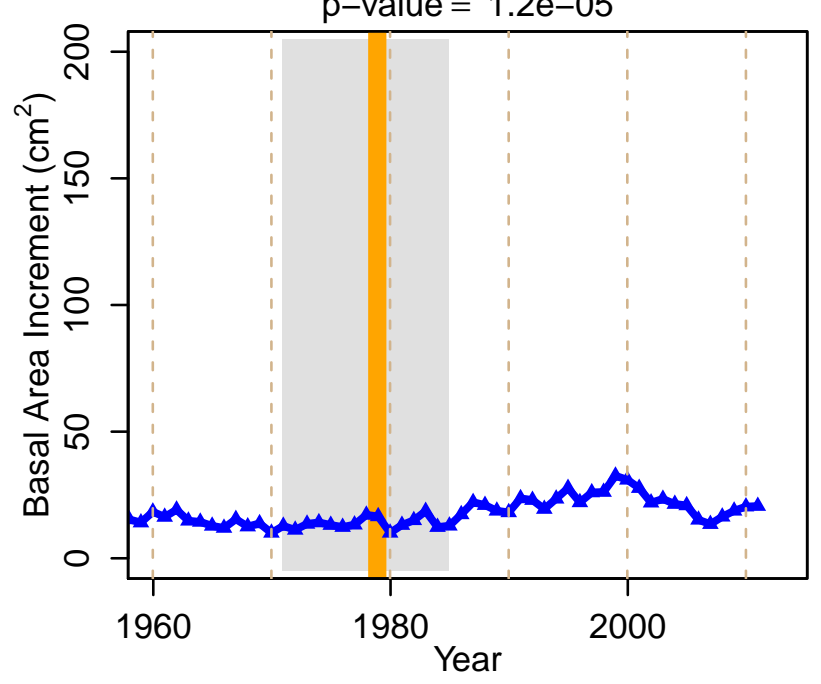

Before $=15.8 \mathrm{~cm}^{2} \mathrm{yr}^{-1} ;$ After $=25.4 \mathrm{~cm}^{2} \mathrm{yr}^{-1}$ $\mathrm{p}-$ value $=3.7 \mathrm{e}-07$

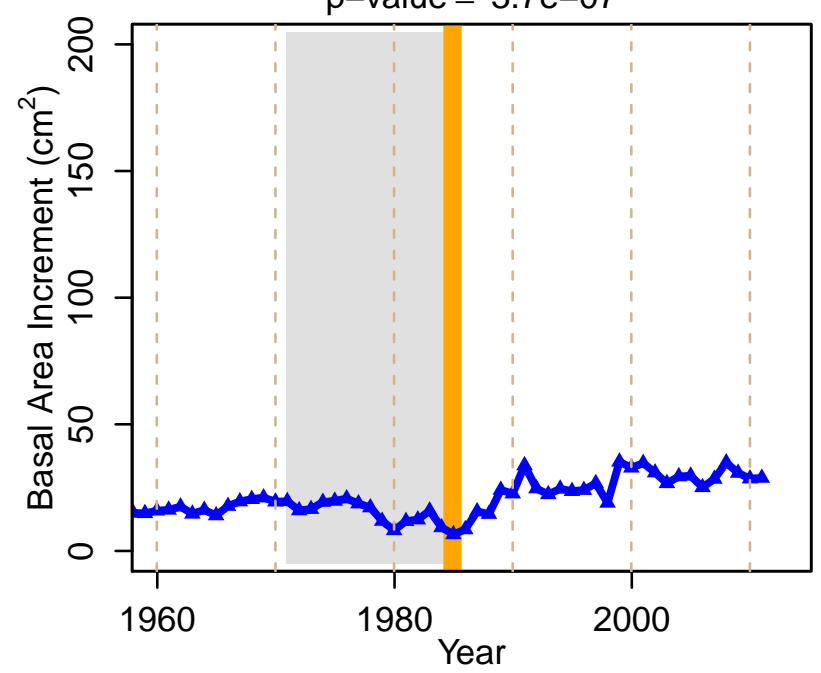




\section{Typical Response}

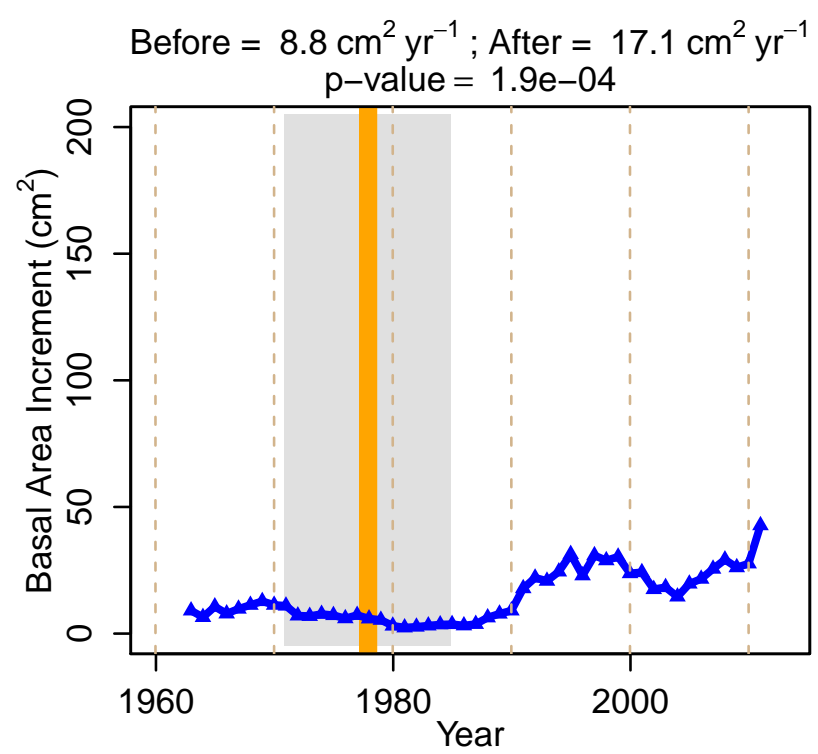

Before $=7.7 \mathrm{~cm}^{2} \mathrm{yr}^{-1} ;$ After $=16.4 \mathrm{~cm}^{2} \mathrm{yr}^{-1}$ $\mathrm{p}$-value $=1.5 \mathrm{e}-12$

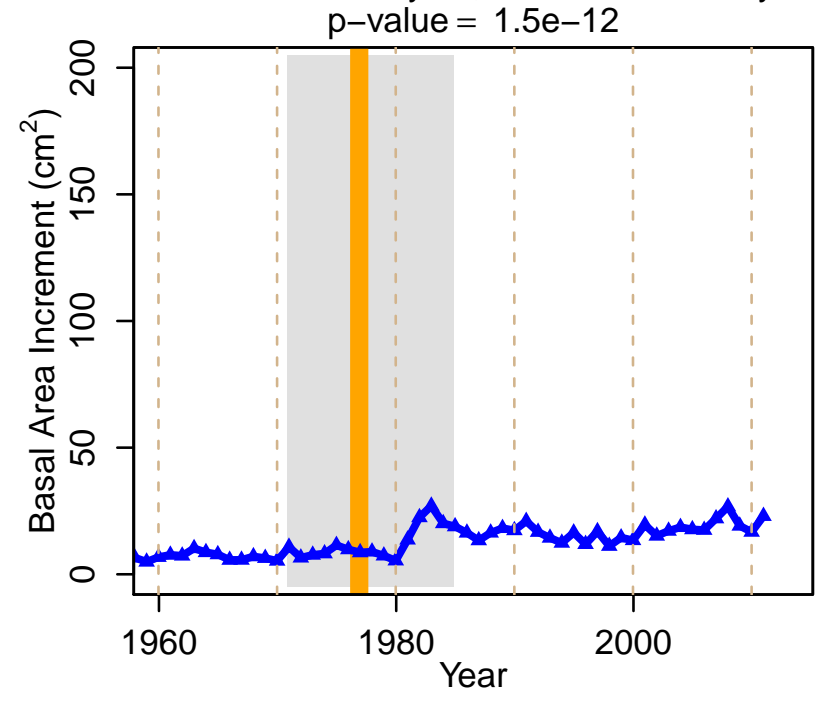

Before $=20.3 \mathrm{~cm}^{2} \mathrm{yr}^{-1} ;$ After $=51 \mathrm{~cm}^{2} \mathrm{yr}^{-1}$ $\mathrm{p}-$ value $=1.2 \mathrm{e}-16$

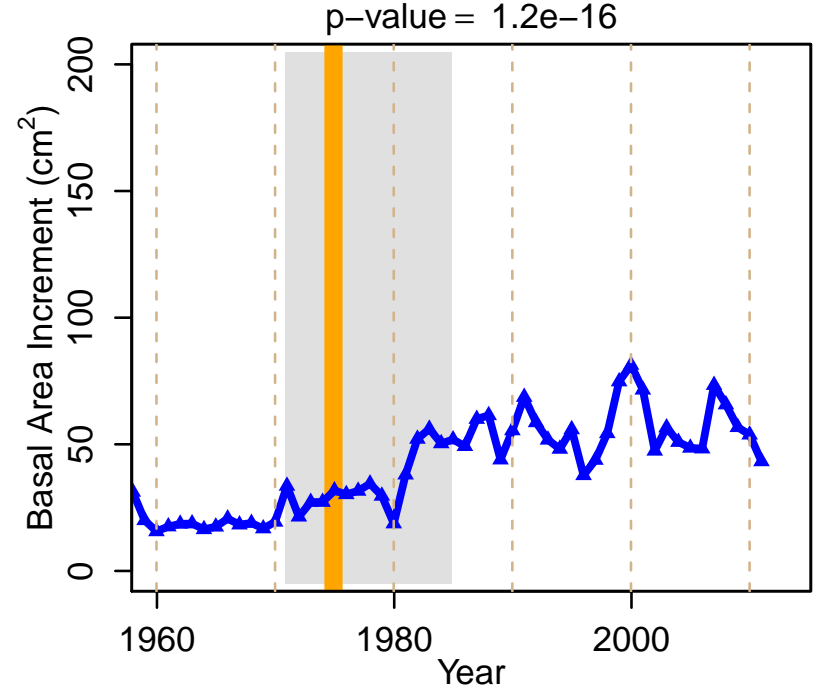

Before $=33.5 \mathrm{~cm}^{2} \mathrm{yr}^{-1} ;$ After $=58.7 \mathrm{~cm}^{2} \mathrm{yr}^{-1}$

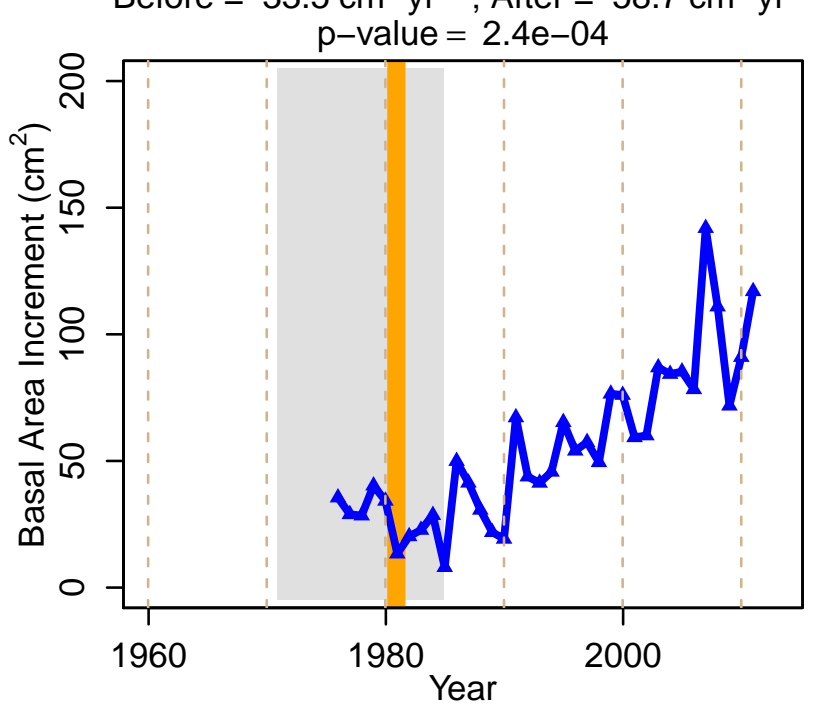

Before $=28.7 \mathrm{~cm}^{2} \mathrm{yr}^{-1} ;$ After $=41.1 \mathrm{~cm}^{2} \mathrm{yr}^{-1}$

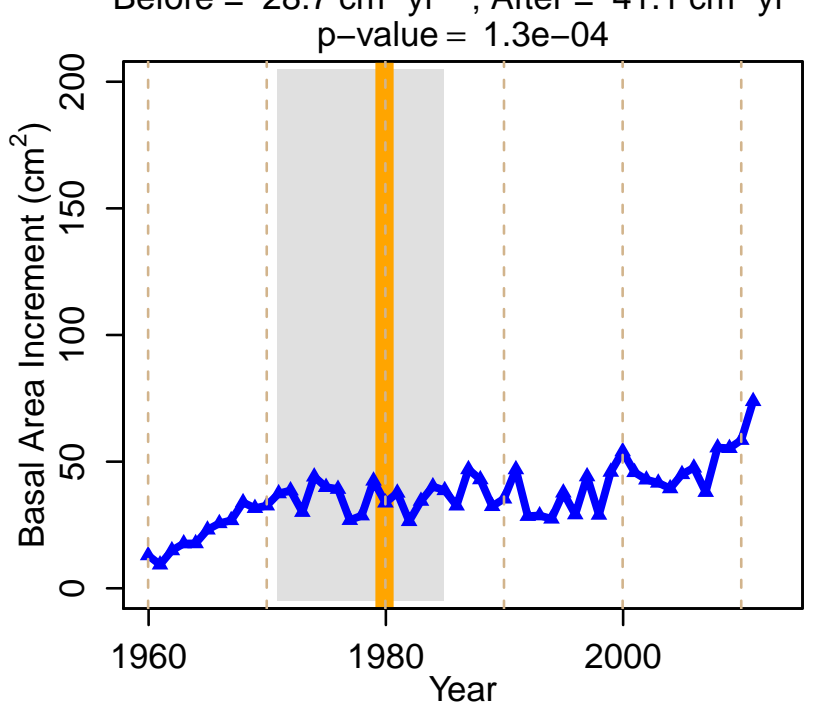

Before $=21.1 \mathrm{~cm}^{2} \mathrm{yr}^{-1} ;$ After $=60.2 \mathrm{~cm}^{2} \mathrm{yr}^{-1}$ $\mathrm{p}$-value $=1.6 \mathrm{e}-14$

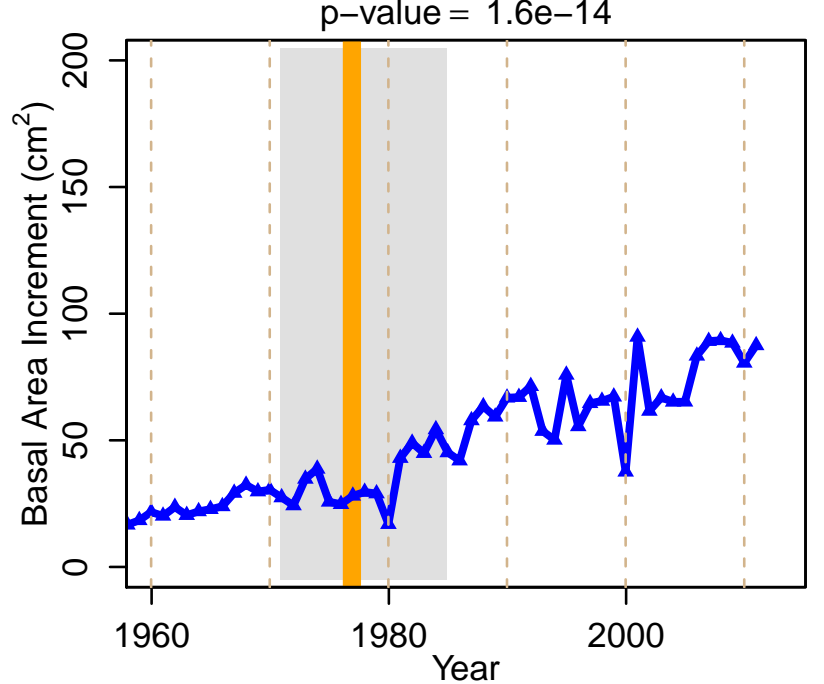

Before $=13.3 \mathrm{~cm}^{2} \mathrm{yr}^{-1} ;$ After $=25.8 \mathrm{~cm}^{2} \mathrm{yr}^{-1}$ $\mathrm{p}-$ value $=5.7 \mathrm{e}-09$

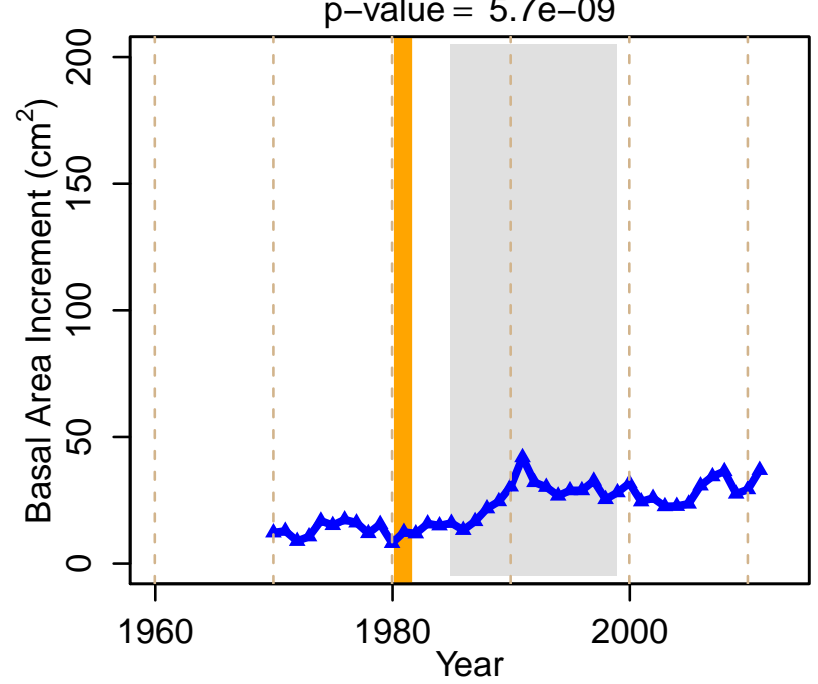

Before $=9.7 \mathrm{~cm}^{2} \mathrm{yr}^{-1} ;$ After $=27.3 \mathrm{~cm}^{2} \mathrm{yr}^{-1}$ $\mathrm{p}-$ value $=1.6 \mathrm{e}-05$

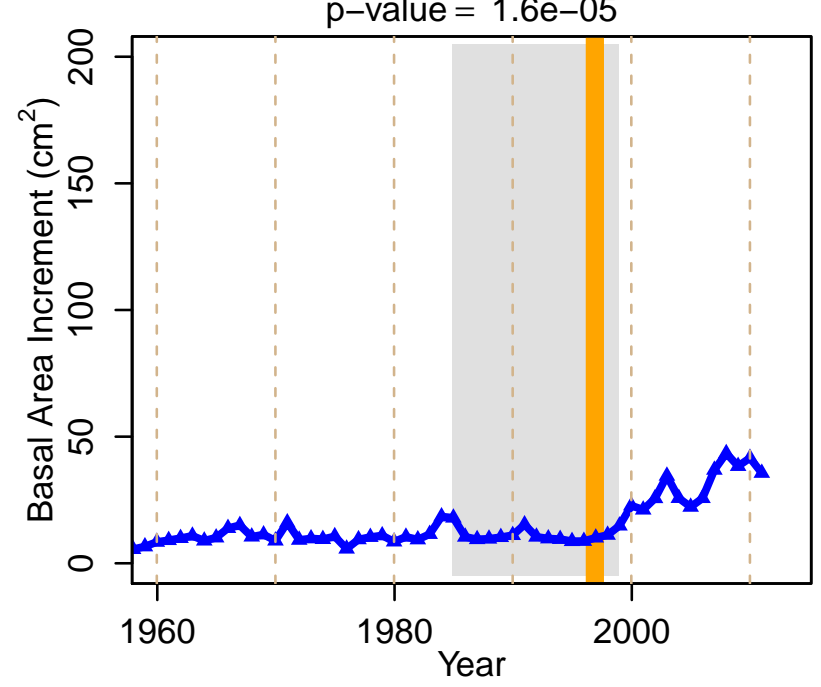

Before $=24 \mathrm{~cm}^{2} \mathrm{yr}^{-1} ;$ After $=44.7 \mathrm{~cm}^{2} \mathrm{yr}^{-1}$ $\mathrm{p}$-value $=2.3 \mathrm{e}-06$

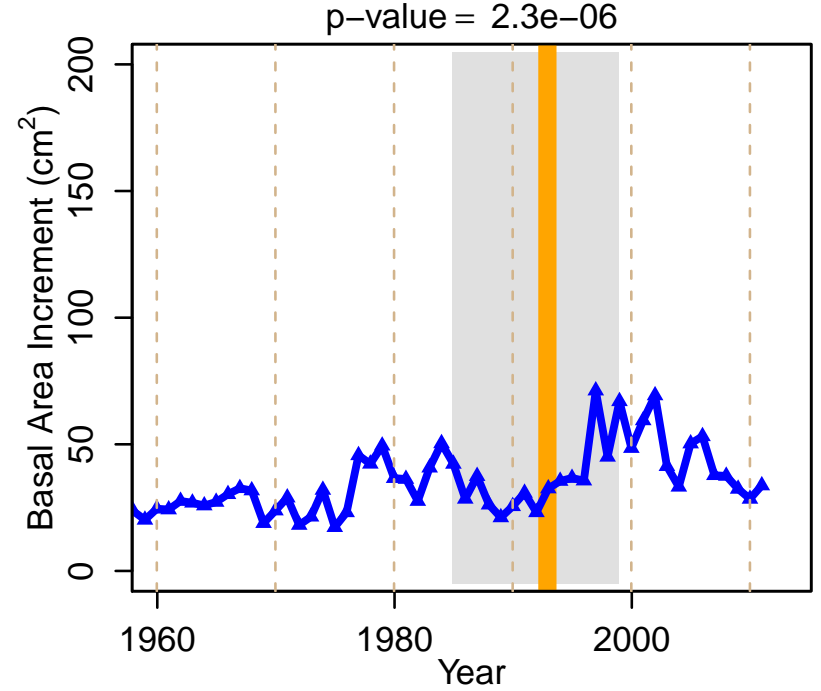




\section{Typical Response}

Before $=20.7 \mathrm{~cm}^{2} \mathrm{yr}^{-1} ;$ After $=34.5 \mathrm{~cm}^{2} \mathrm{yr}^{-1}$ $\mathrm{p}$-value $=1.9 \mathrm{e}-07$

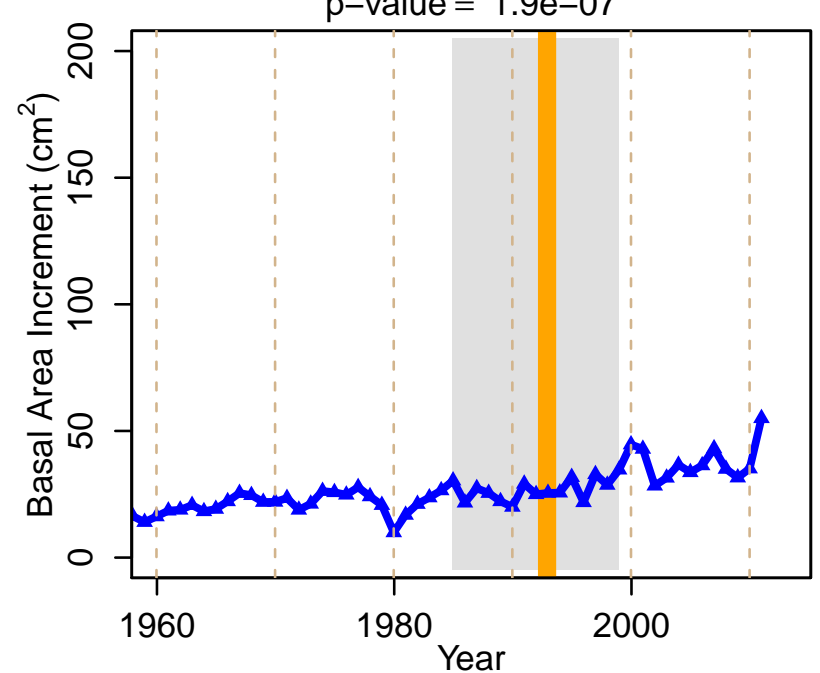

Before $=25.7 \mathrm{~cm}^{2} \mathrm{yr}^{-1} ;$ After $=37.2 \mathrm{~cm}^{2} \mathrm{yr}^{-1}$ $\mathrm{p}-$ value $=6.7 \mathrm{e}-05$

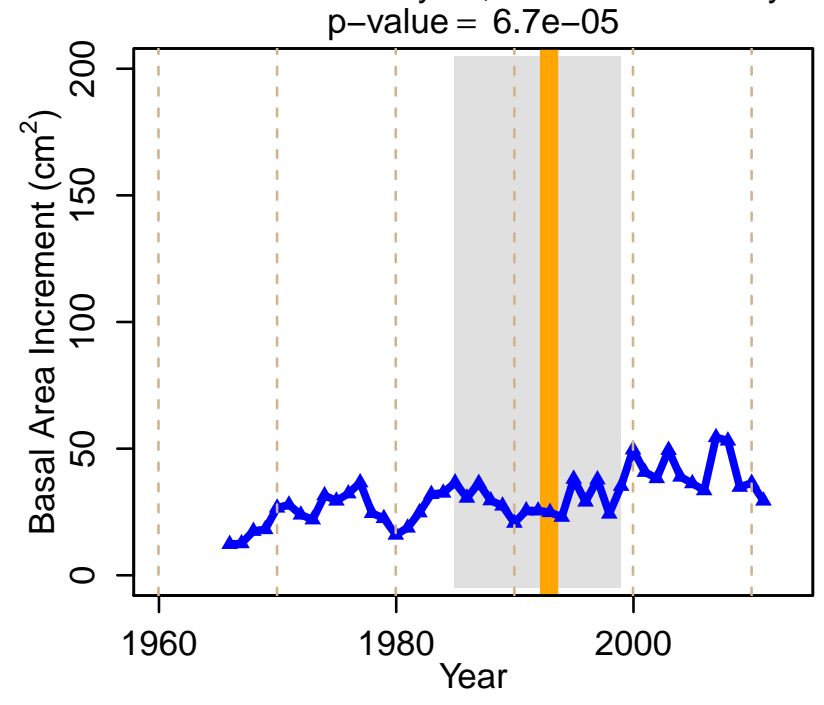

Before $=12.4 \mathrm{~cm}^{2} \mathrm{yr}^{-1} ;$ After $=33.4 \mathrm{~cm}^{2} \mathrm{yr}^{-1}$ $\mathrm{p}$-value $=2.5 \mathrm{e}-09$

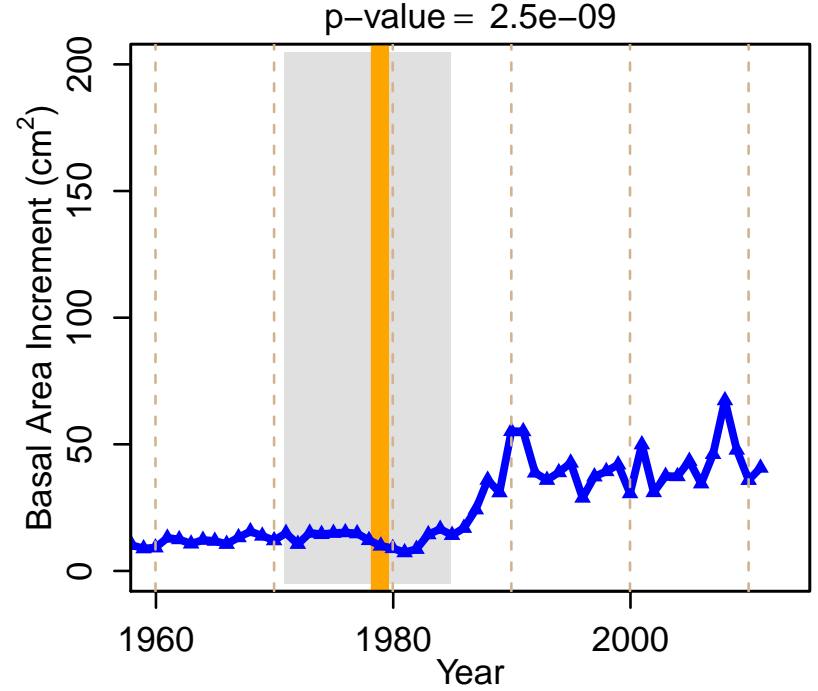

Before $=8.8 \mathrm{~cm}^{2} \mathrm{yr}^{-1} ;$ After $=17.9 \mathrm{~cm}^{2} \mathrm{yr}^{-1}$ $\mathrm{p}-$ value $=1.5 \mathrm{e}-13$

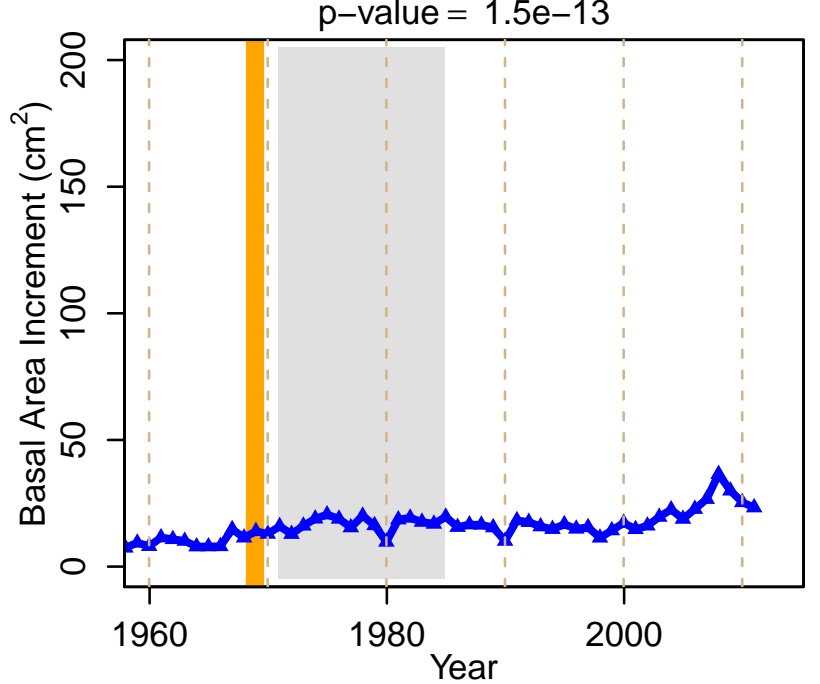

Before $=5.9 \mathrm{~cm}^{2} \mathrm{yr}^{-1} ;$ After $=10.8 \mathrm{~cm}^{2} \mathrm{yr}^{-1}$

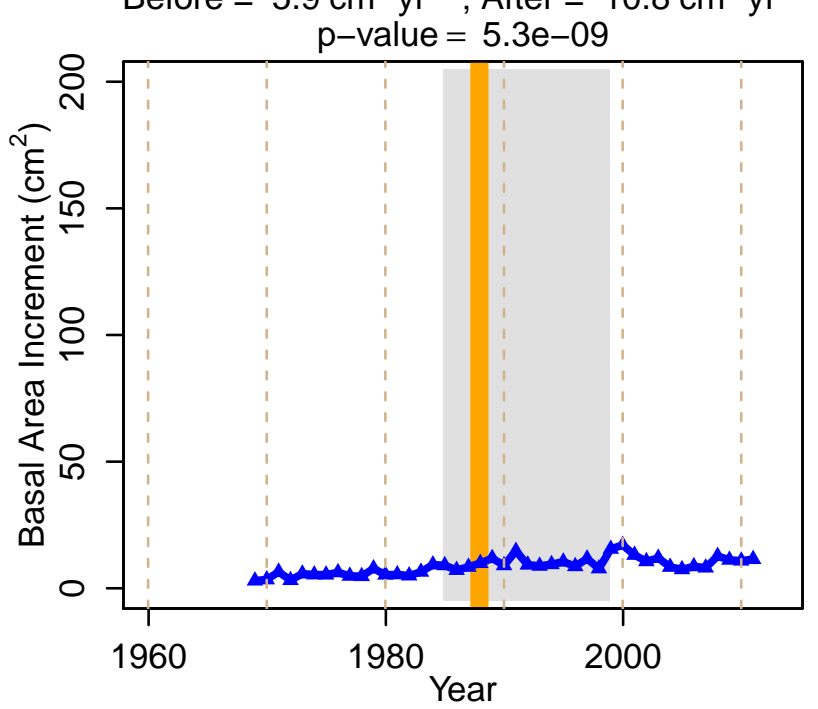

Before $=31.2 \mathrm{~cm}^{2} \mathrm{yr}^{-1} ;$ After $=82.1 \mathrm{~cm}^{2} \mathrm{yr}^{-1}$ $\mathrm{p}-$ value $=2.1 \mathrm{e}-13$

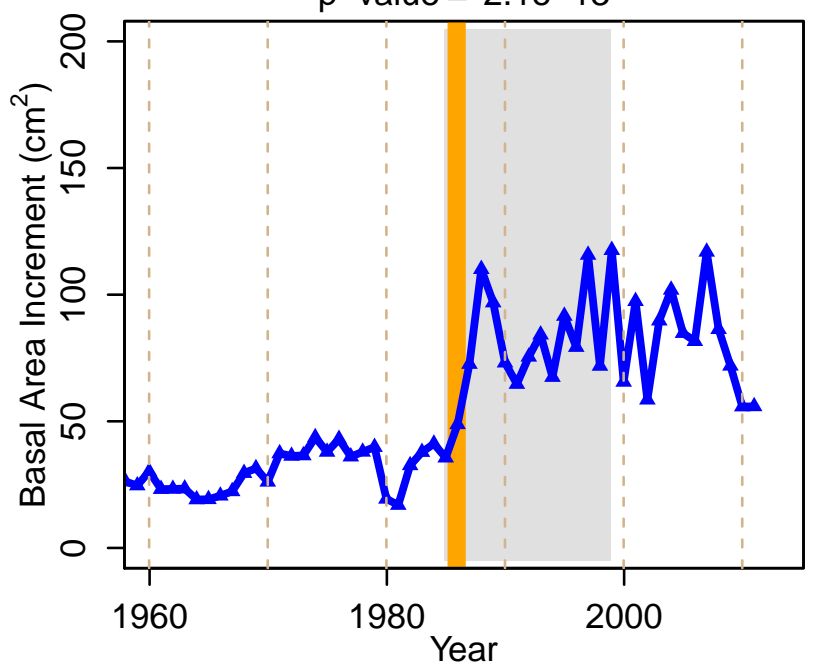

Before $=10.4 \mathrm{~cm}^{2} \mathrm{yr}^{-1} ;$ After $=26.6 \mathrm{~cm}^{2} \mathrm{yr}^{-1}$ $\mathrm{p}$-value $=4 \mathrm{e}-17$

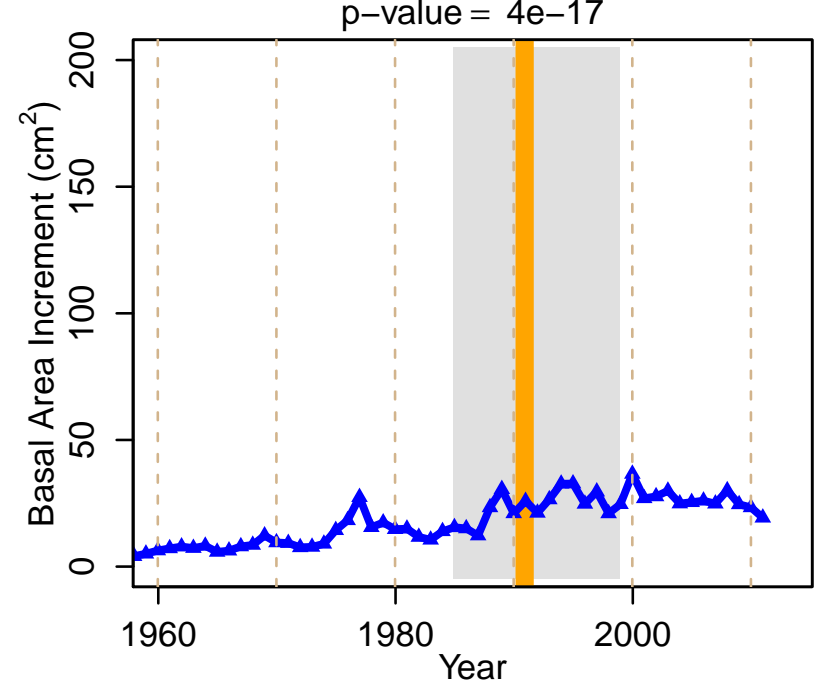

Before $=9.5 \mathrm{~cm}^{2} \mathrm{yr}^{-1} ;$ After $=12.8 \mathrm{~cm}^{2} \mathrm{yr}^{-1}$ $\mathrm{p}-$ value $=6.1 \mathrm{e}-08$

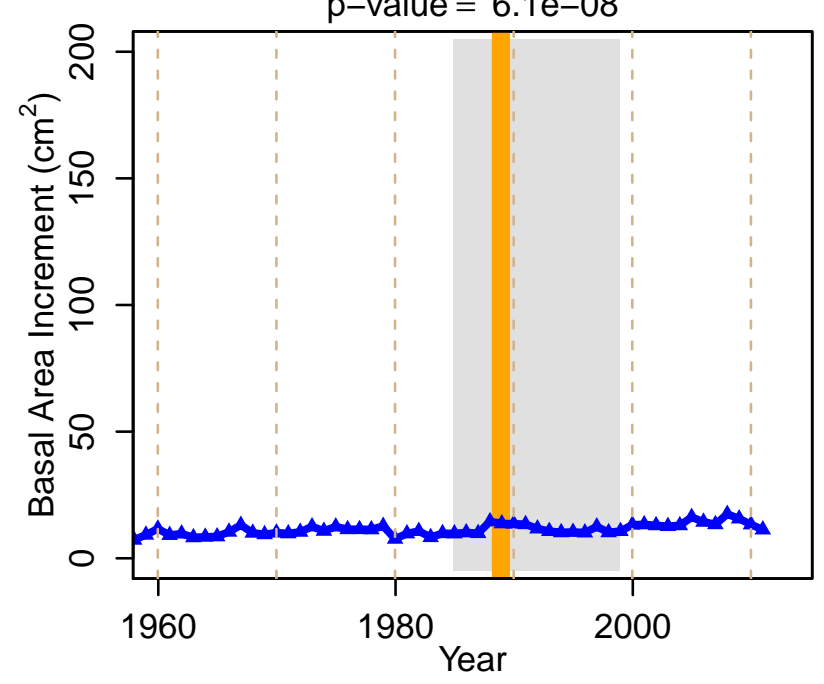




\section{Negative/No Response}

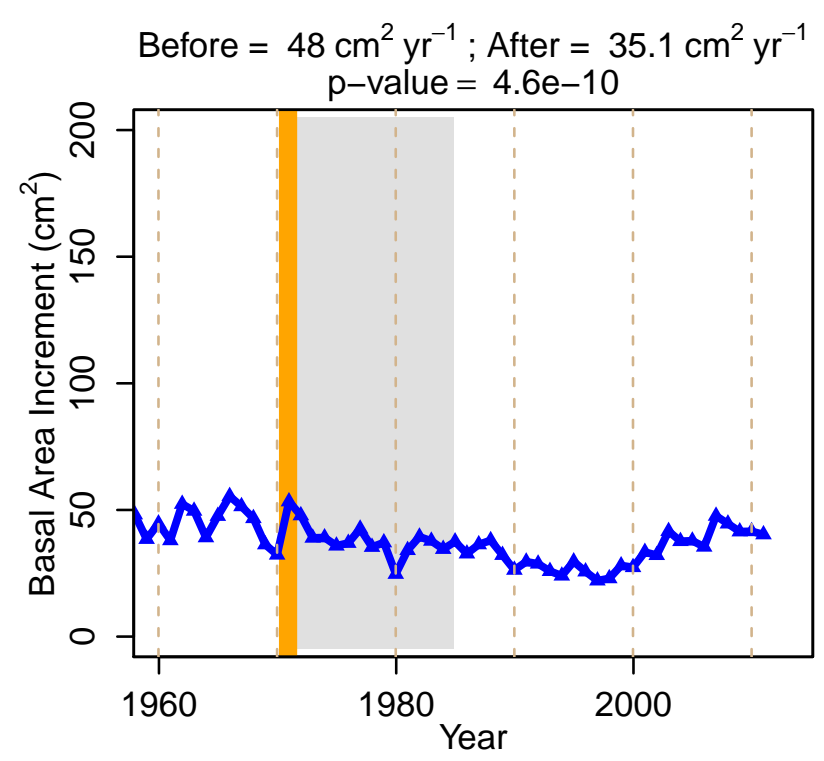

Before $=25.2 \mathrm{~cm}^{2} \mathrm{yr}^{-1} ;$ After $=29.2 \mathrm{~cm}^{2} \mathrm{yr}^{-1}$ $\mathrm{p}$-value $=0.23$

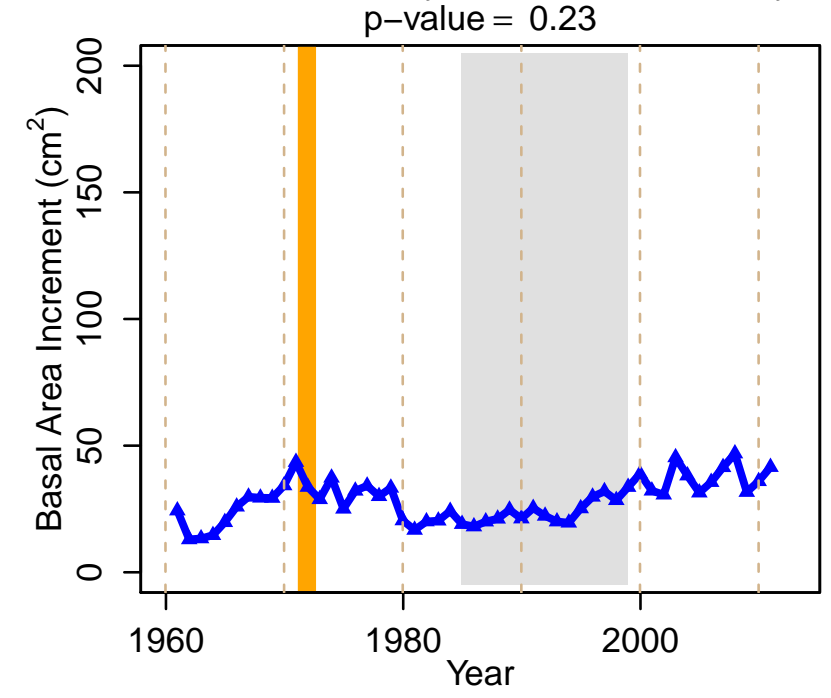

Before $=35.1 \mathrm{~cm}^{2} \mathrm{yr}^{-1} ;$ After $=21.3 \mathrm{~cm}^{2} \mathrm{yr}^{-1}$

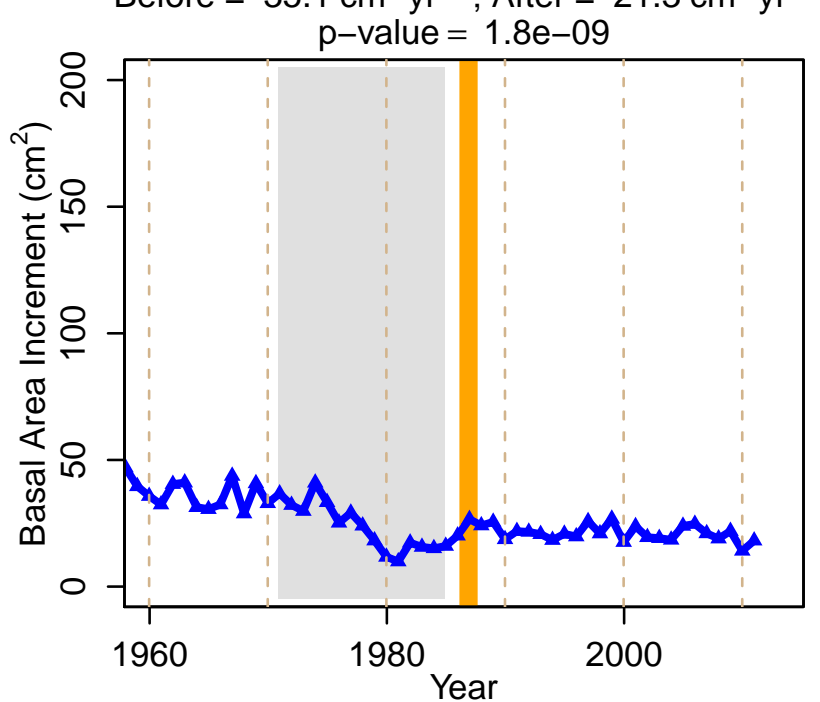

Before $=20.8 \mathrm{~cm}^{2} \mathrm{yr}^{-1} ;$ After $=23.1 \mathrm{~cm}^{2} \mathrm{yr}^{-1}$ $\mathrm{p}$-value $=0.19$

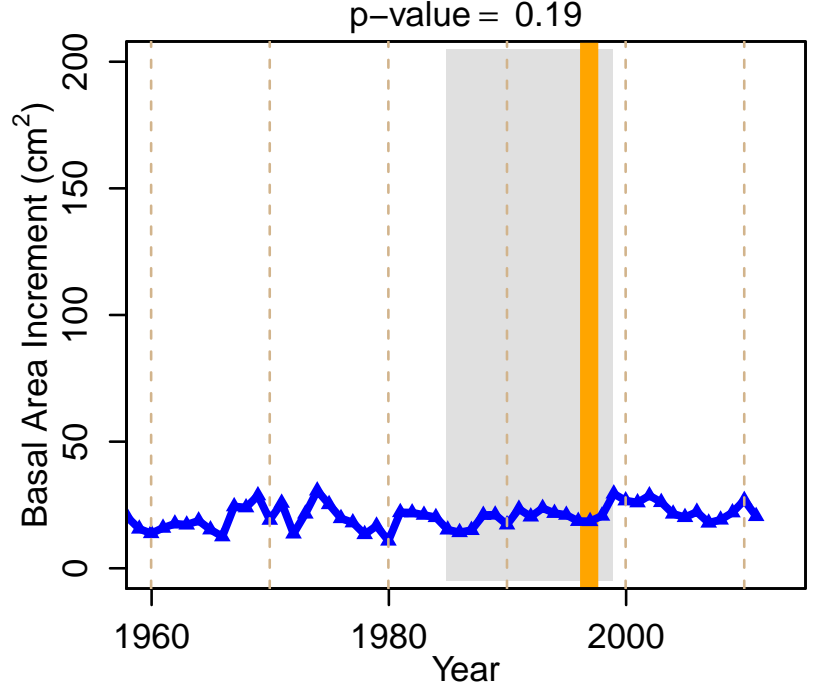

Before $=37.3 \mathrm{~cm}^{2} \mathrm{yr}^{-1} ;$ After $=27.8 \mathrm{~cm}^{2} \mathrm{yr}^{-1}$ $\mathrm{p}-$ value $=1.3 \mathrm{e}-02$

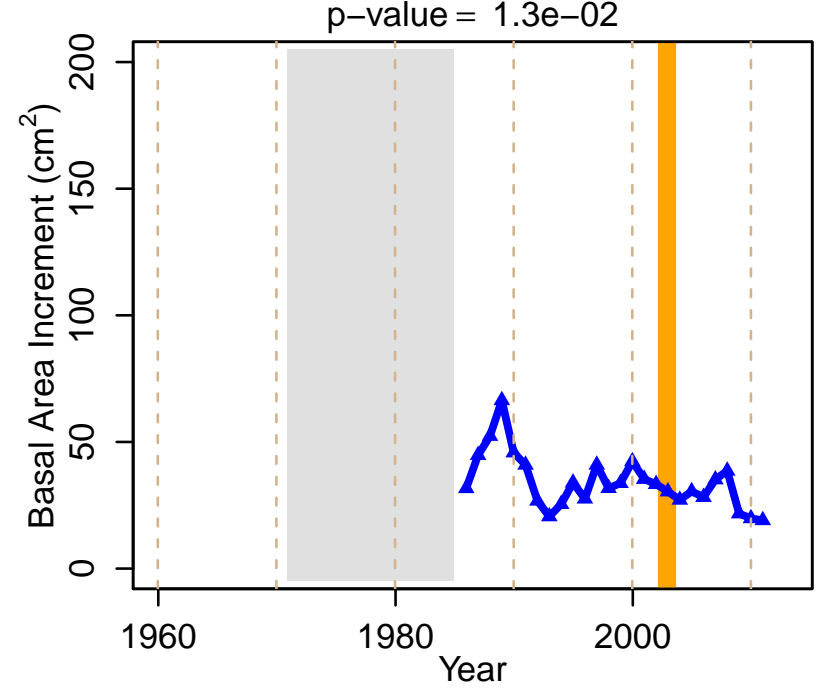

Before $=58.6 \mathrm{~cm}^{2} \mathrm{yr}^{-1} ;$ After $=52.8 \mathrm{~cm}^{2} \mathrm{yr}^{-1}$ $\mathrm{p}-$ value $=0.33$

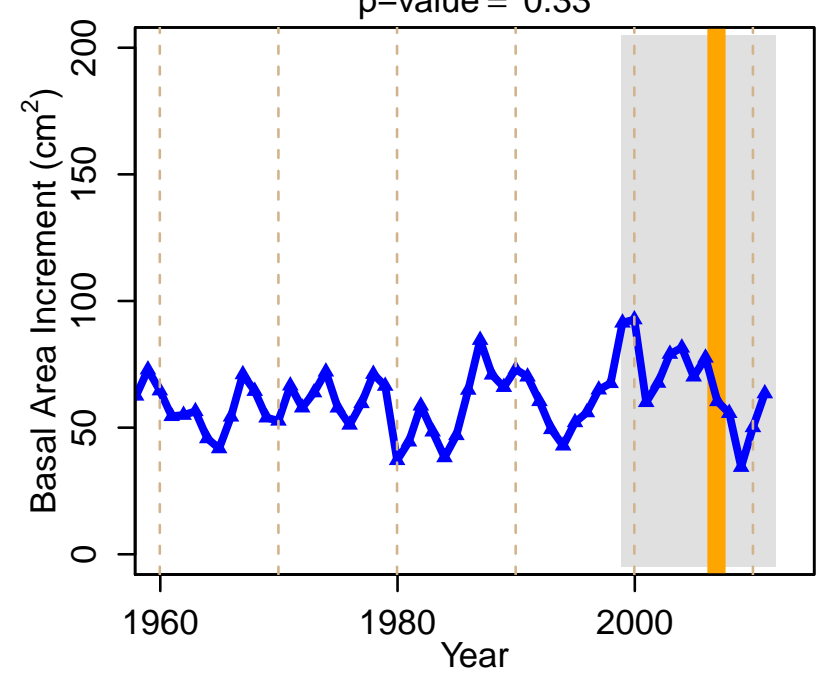




\section{Stable Urban}
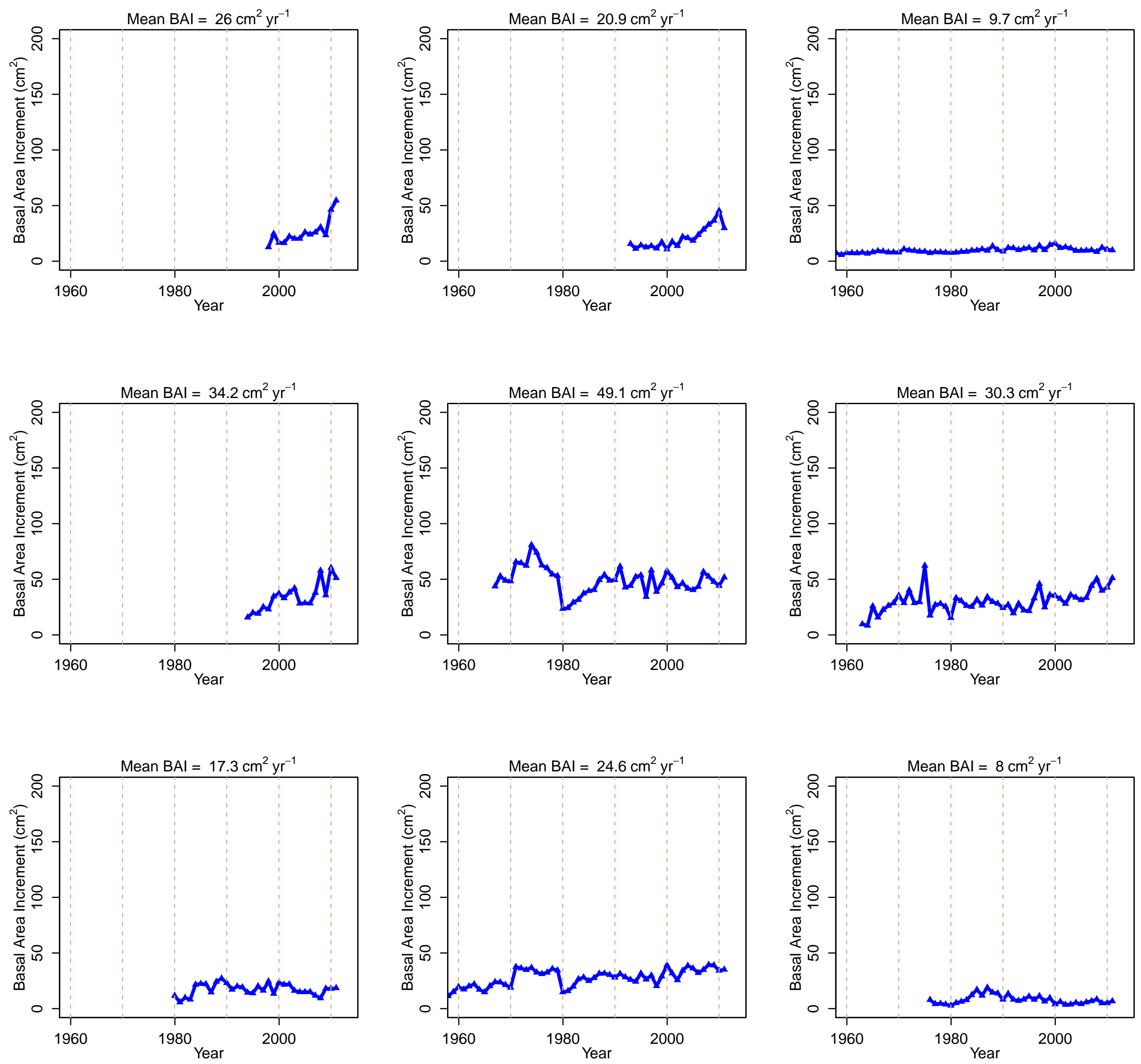


\section{Stable Urban}
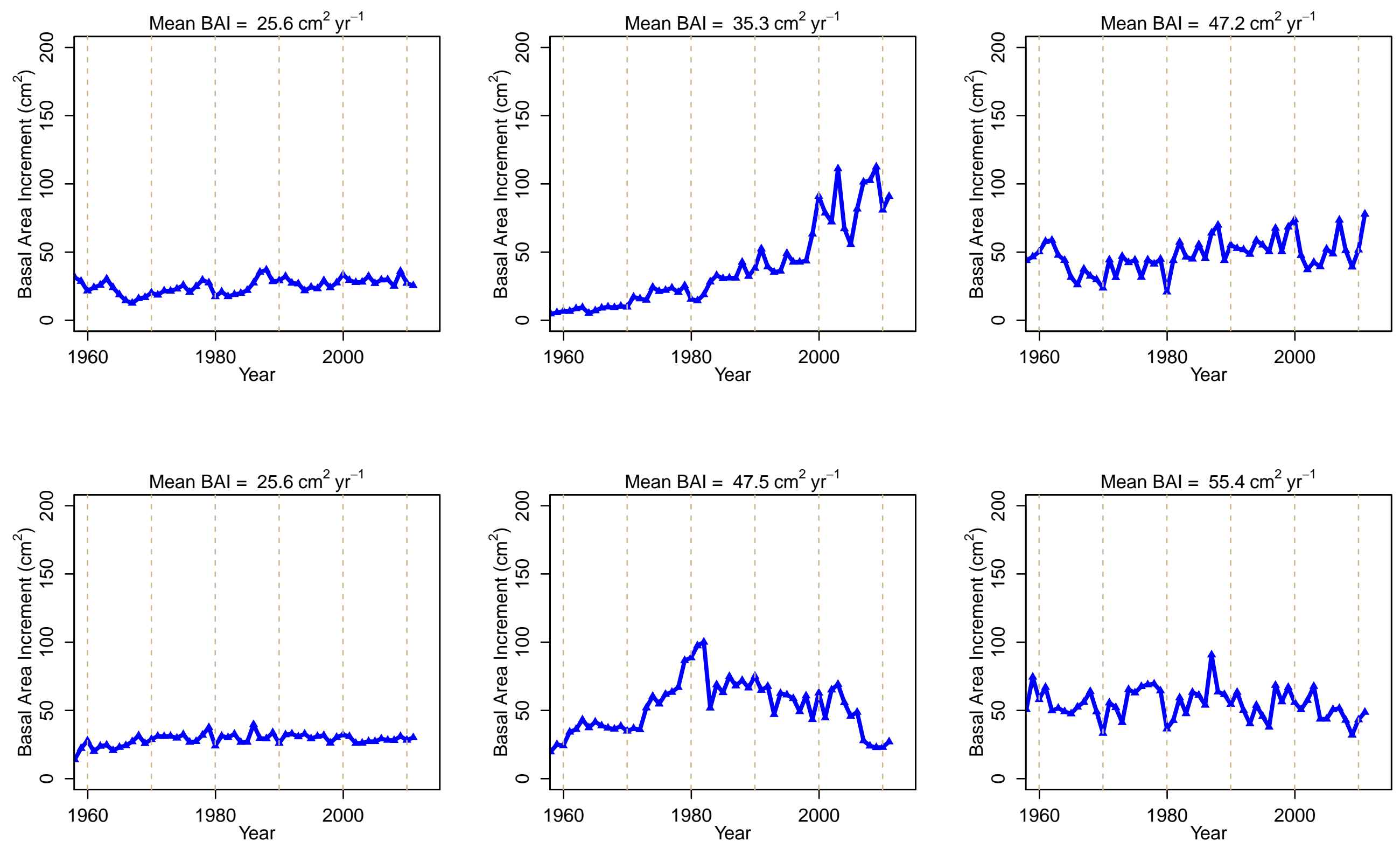


\section{Boston Street Trees}
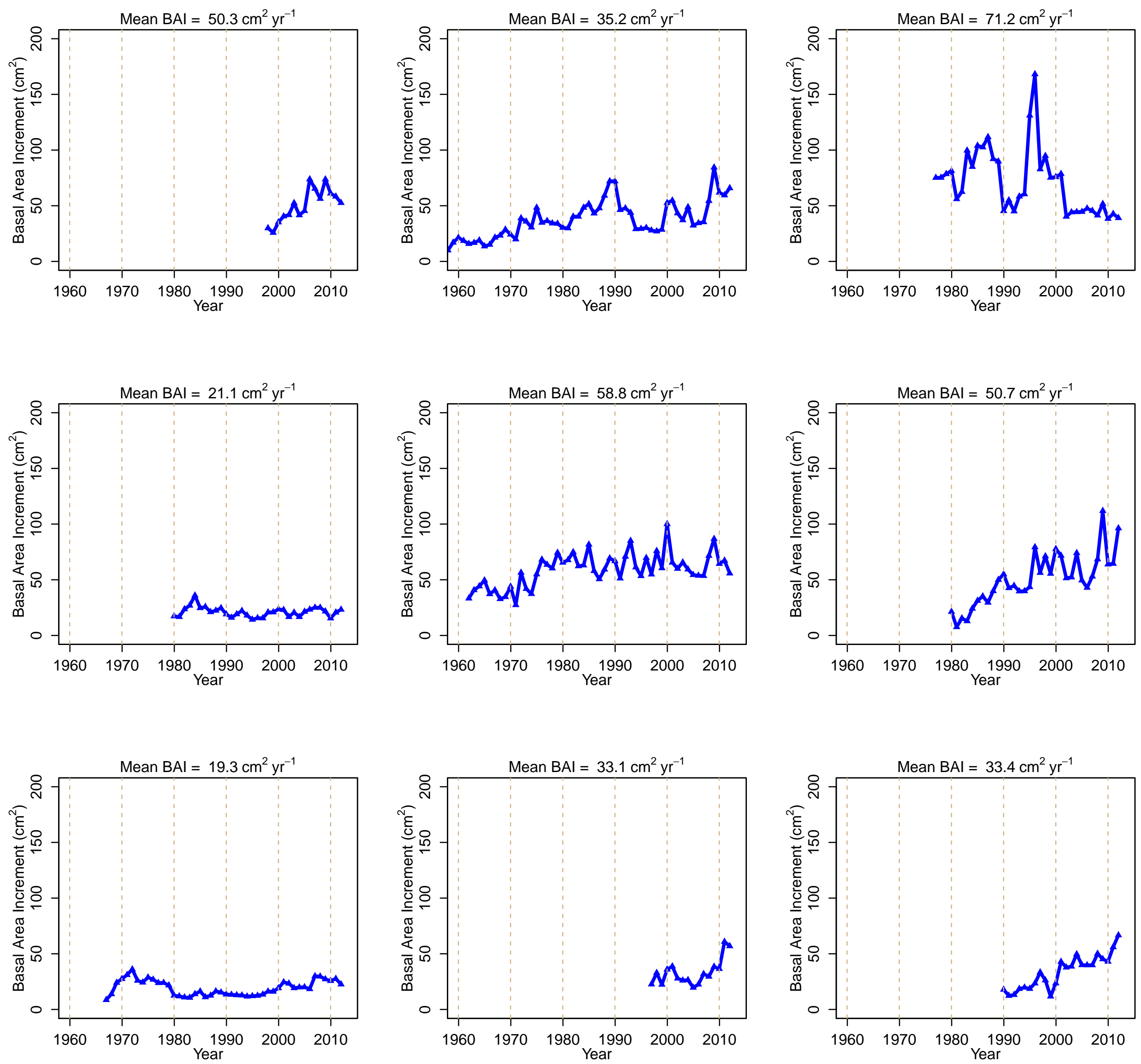


\section{Boston Street Trees}
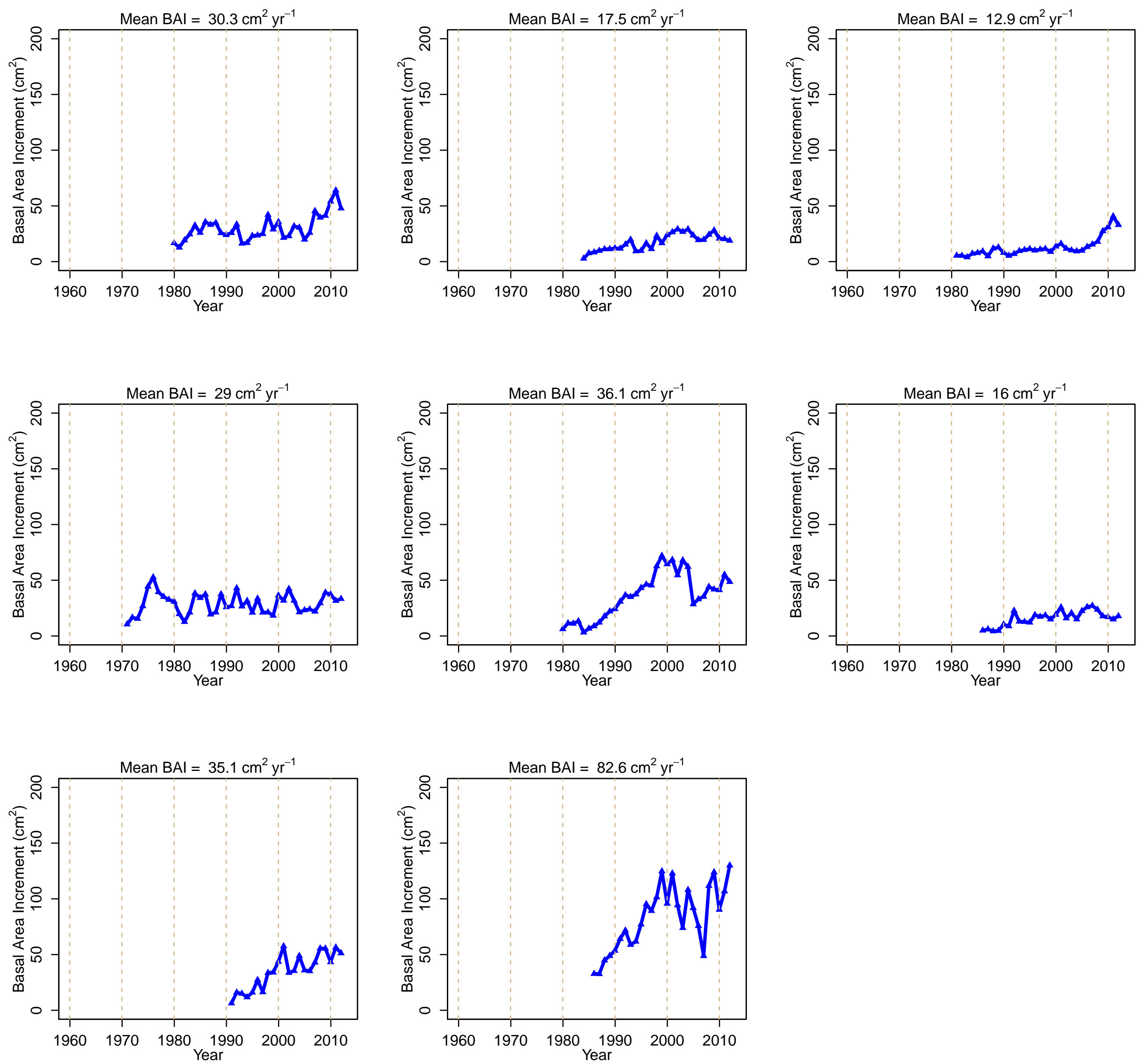